\title{
Fine Mapping of Cla015407 Controlling Plant Height in Watermelon
}

\author{
Taifeng Zhang, Jiajun Liu, Sikandar Amanullah, Zhuo Ding, Haonan Cui, Feishi Luan, and \\ Peng Gao \\ College of Horticulture and Landscape Architecture, Northeast Agricultural University, Harbin, \\ Heilongjiang 150030, China; and Key Laboratory of Biology and Genetic Improvement of \\ Horticulture Crops (Northeast Region), Ministry of Agriculture and Rural Affairs, Harbin, \\ Heilongjiang 150030, China
}

\begin{abstract}
AdDitional INDEX wORDs. bulked segregant analysis, candidate gene, Citrullus lanatus, cleaved amplified polymorphism sequence, dwarfism, GA 3 $\beta$-hydroxylase, genetic analysis, kompetitive allele-specific polymerase chain reaction

ABstract. The plant compact and dwarf growth habit is an important agronomic trait when breeding watermelon (Citrullus lanatus) cultivars because of their reduced vine length, high-density planting, and better land utilization; however, the genetic basis of the dwarf growth habit is not well-known. In this study, the plant population of six generations, P1, P2, F1, F2, BC1P1, and BC1P2, were studied. A genetic segregation analysis demonstrated that dwarfism is mainly controlled by a single recessive $C l d w$ gene. Furthermore, whole-genome sequencing of two distinct watermelon cultivars, W1-1 (P1) and 812 (P2), was performed and preliminarily mapped through a bulked segregant analysis of $\mathrm{F} 2$ individuals that revealed the $C l d w$ gene locus on chromosome 9. Two candidate genes, $\mathrm{Cla015407}$ and Cla015408, were discovered at the delimited region of $43.2 \mathrm{~kb}$ by fine mapping, and gene annotation exposed that the Cla015407 gene encodes gibberellic acid $3 \beta$-hydroxylase protein. In addition, a comparative analysis of gene sequence and cultivars sequences across the reference genome of watermelon revealed the splice site mutation in the intron region of the $C l d w$ gene in dwarf-type cultivar 812. The quantitative real-time polymerase chain reaction exhibited a significantly higher expression of the $\mathrm{Cla015407}$ gene in cultivar W1-1 compared with 812. There was no significant difference in the vine length of both cultivars after gibberellic acid treatment. In brief, our fine mapping demonstrated that $\mathrm{Cla015407}$ is a candidate gene controlling dwarfism of watermelon plants.
\end{abstract}

Watermelon (Citrullus lanatus) is an important cucurbit crop that covers $7 \%$ of the total vegetable crop production area (Guo et al., 2012). The dwarf-type plants have significant advantages in agricultural crops because of their high-density planting, resistance to wind storms, lodging condition tolerance, and reduced major losses during mechanical harvesting operations (Amasino et al., 2003).

Numerous dwarfism-associated traits such as short internodes, reduced main stem length, and bushy-type growth habits of different crops have been studied to identify the candidate genes controlling the compact and dwarf growth habit. It was reported that the compact and dwarf growth habit of watermelon is controlled by allelism of two genes, $d w-1$ and $d w-1 s$, and three independent loci, $d w-2, d w-3$, and $d w-4$ (Huang et al., 1998; Loy and Liu, 1972; Yang et al., 2009). The first recessive gene, $d w-1$, was discovered in dwarf-type watermelon; it causes mutation in the standard-type watermelon cultivar WB-2 by synthesis of abnormal internodal cells (short internodes) (Mohr, 1956). The second genetically inherited recessive gene, $d w-1 s$, was detected in short-vine watermelon cultivar Somali Local (Dyutin and

Received for publication 19 Apr. 2020. Accepted for publication 1 Mar. 2021

Published online 3 May 2021.

We sincerely acknowledge the editors and reviewers for their valuable comments that have helped to improve this manuscript.

This work was supported by the National Nature Science Foundation of China (no. 31672177), and "Academic Backbone" Project of Northeast Agricultural University (no. 16XG06).

P.G. is the corresponding author. E-mail: gaopeng neau@163.com.

This is an open access article distributed under the CC BY-NC-ND license (https://creativecommons.org/licenses/by-nc-nd/4.0/).
Afanas'eva, 1987). The third recessive gene, $d w$-2, was identified in the short-internode vine cultivar Bush Desert King (BDK), which has shortened internode cells, and the fourth gene, $d w-3$, was discovered in the dwarf male-sterile watermelon cultivar DMSW (Huang et al., 1995). The gene $d w-4$ was identified in a self-pollinated population of short-vine watermelon cultivar 5-6y (Yang et al., 2009). Recently, potential candidate genes encoding gibberellic acid (GA) 20-oxidase-like protein and controlling dwarfism have been mapped on watermelon chromosome 7 (Dong et al., 2018). Moreover, the dwarfism gene $C l d w-1$ encoding an ATP-binding cassette transporter (ABC transporter)-related protein was also identified in watermelon line WM102 on chromosome 9 (Zhu et al., 2019).

Many genes controlling the compact and dwarf growth habit have been mapped in few cucurbit crops, and those findings strongly indicated the differential causes of the plant compact and dwarf growth habit. Regarding cucumber (Cucumis sativus), a dwarf-type mutant was obtained from cucumber 'Lemon' because of the mutagenesis resulting from the $d w$ gene (Robinson and Mishanec, 1965). Then, the recessive gene $c p$ was identified in cucumber line PI308915, which causes a shorter internodal length and controls the angle between leaves and side branches of compact plants (Kauffman and Lower, 1976). A compact mutant, $c p-2$, was also identified in ethyl methanesulfonate-treated cucumber plants, but the allelism of both $c p$ and $c p-2$ was not completely understood (Kubicki et al., 1986). Then, the mutant gene $c p$ was also identified in a short-vine cucumber cultivar and fine-mapped at the $220-\mathrm{kb}$ genetic region located at chromosome 4, which is highly homologous with cytokinin oxidase (CKX) (Li et al., 2011). Regarding melon (Cucumis melo), three recessive genes, $s i-1, s i-2$, and $s i-3$, have been reported to control 
the compactness or dwarfism of three comparative cultivars, Maindwarf, Persia 202, and UC Top Mark bush, respectively (Knavel, 1988, 1990; Paris et al., 1984). Among these genes, the si-1 gene is mainly connected with yellow virescence (Pitrat, 1991) and displays bushy-type plant phenotypes with compact growth as well as a short internodal length. Furthermore, the $m d w 1$ gene was identified on a 1.8-cM region in the dwarf-type mutant line PNU-WT1, which exhibited high homology with erecta and ubiquitin (Hwang et al., 2014). During a recent study of melon, a putative genetic region associated with short internodal length was mapped on chromosome 7, and gene annotation predicted erecta as a recessive gene for controlling dwarfism (Zhang et al., 2019).

The crop morphological traits are regulated by multiple genes, and the compact and dwarf growth habit genes are mostly involved in biosynthesis or signal transduction pathways of plant hormones. Among these types of hormone, GAs are the most widely studied hormones (Nagai et al., 2018; Peng, 1999) because dwarf-type plants are mostly divided into the GA-deficient type or insensitive type based on the location of their mutant genes during hormonal biosynthesis or metabolic pathways. To date, numerous genes encoding GA synthesis-related enzymes have been cloned (Chiang et al., 1995; Sun, 1992; Winkler, 1995) and expressed as mutant genes in different plant types (Hedden and Phillips, 2001). Mainly, GA 3ß-hydroxylase catalyzes the absolute activity in the GA biosynthesis pathway (Yamaguchi, 2008), and its pivotal role in maize (Zea mays) and rice (Oryza sativa) has been reported (Chen et al., 2014; Itoh et al., 2001; Teng et al., 2013).

The bulked segregant analysis (BSA) is a rapid technique for mapping genes related to the measured phenotype of different crops and identifying the tightly closed genetic markers linked to the major genes and that phenotype. In 1991, the BSA was primarily used to map disease resistance genes (Michelmore et al., 1991). It has been widely used for rapid gene mapping and the identification of genetic markers linked with quantitative or qualitative traits of interest in horticultural crops such as watermelon (Dong et al., 2018), melon (Li et al., 2017; Liu et al., 2019), cucumber (Zhang et al., 2015), and tomato [Solanum lycopersicum (Zhao et al., 2016)].

During this study, whole-genome sequencing and BSA were performed for primary and fine mapping of genes controlling dwarfism. In addition, cleaved amplified polymorphism sequence (CAPS) and kompetitive allele-specific polymerase chain reaction (KASP) markers were developed and used. The genetic inheritance analysis was also performed by using plant populations of six generations. The present study results will provide additional insight for marker-assisted breeding of dwarf watermelon cultivars in China.

\section{Materials and Methods}

Plant materials and population development. Two distinct watermelon cultivars, W1-1 and 812, were selected as plant materials based on their distinguished plant characteristics. The cultivar W1-1 is a female with standard-type vine length and moderate internodes, whereas cultivar 812 is a male with a dwarf-type bushy vine length and short internodes (Fig. 1). These distinct cultivars were cross-pollinated to develop the further generations $\left(\mathrm{F}_{1}, \mathrm{~F}_{2}, \mathrm{BC}_{1} \mathrm{P} 1\right.$, and $\left.\mathrm{BC}_{1} \mathrm{P} 2\right)$; they were also used for whole-genome resequencing, $\mathrm{BSA}$, and genetic

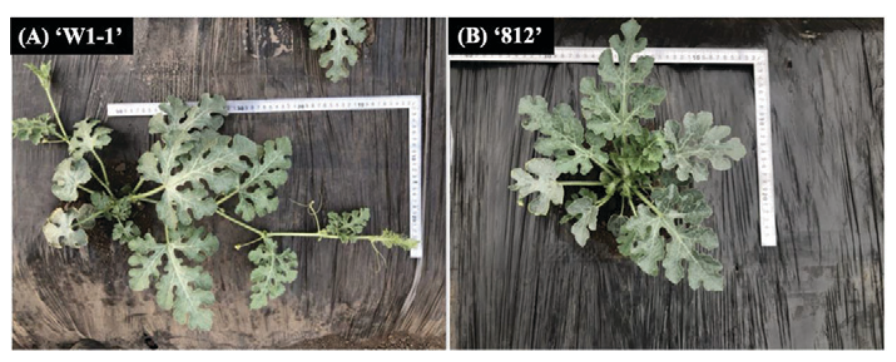

Fig. 1. Differences in the plant morphological characteristics of two distinct watermelon cultivars: (A) $35 \mathrm{~d}$ after sowing W1-1 (P1, standard type) grown in the green house and (B) $35 \mathrm{~d}$ after sowing 812 (P2, dwarf type) grown in a greenhouse.

inheritance analyses. All experiments were continued over 2 years (2018 and 2019) in a plastic green house at XiangYang Experimental Farm, Northeast Agricultural University, Harbin, China (lat. $44^{\circ} 04^{\prime} \mathrm{N}$, long. $125^{\circ} 42^{\prime} \mathrm{E}$ ). The standard horticultural practices were also performed according to the needs and symptoms of plant cultivation, plant growth, and typical climatic conditions.

In 2018, the watermelon cultivars were grown and crossed to acquire their $F_{1}$ hybrid. Then, a single $F_{1}$ hybrid was further self-crossed and back-crossed to produce the $\mathrm{F}_{2}, \mathrm{BC}_{1} \mathrm{P} 1$, and $\mathrm{BC}_{1} \mathrm{P} 2$ generations separately. For plant cultivation, seedlings of all generations were germinated in small polyethylene bags filled with an organic mixture of soil and peat moss; then, well-developed seedlings at the fully expanded leaf stage were transferred to ridges in the plastic greenhouse with a row space of $50 \mathrm{~cm}$ and plant space of $12 \mathrm{~cm}$.

The plant phenotypes were visually observed at the seedling stage and plant reproductive stage and classified as the standard type or dwarf type based on their main stem length (MSL). When the MSL of all plants was $\approx 50 \mathrm{~cm}$ long, the side stems were trimmed and the data of the main stem (still attached to the plant) of the plant population of six generations were recorded on same day by using a simple measuring tape. The genetic inheritance analysis was performed by investigating the segregation ratio of standard-type and dwarf-type plant phenotypes of six generations, $\mathrm{P} 1(\mathrm{n}=15), \mathrm{P} 2(\mathrm{n}=15), \mathrm{F}_{1}(\mathrm{n}=15), \mathrm{F}_{2}(\mathrm{n}=$ $196), \mathrm{BC}_{1} \mathrm{P} 1(\mathrm{n}=150)$, and $\mathrm{BC}_{1} \mathrm{P} 2(\mathrm{n}=150)$. Furthermore, a total of 184 of $196 \mathrm{~F}_{2}$ individuals were used for primary mapping. In 2019, a total of $1439 \mathrm{~F}_{2}$ individuals were grown according to the same planting geometry and trait-measuring methods used during 2018. The fine-mapping region and marker-trait association were obtained from total of 1623 (184 and 1439) $F_{2}$ individuals.

WhOLE-GenOME RESEQUENCING OF BULKED DNA. Total DNA was isolated by using the improved cetyltrimethylammonium ammonium bromide (CTAB) technique (Allen et al., 2006). The DNA concentration was measured by using a spectrophotometer (SMA3000; Plextech, Shenzhen, China). DNA purity was checked using 1\% agarose gel electrophoresis. A total of two bulked samples were arranged by mixing an equal proportion of extracted DNA from 30 standard-type and 30 dwarf-type $F_{2}$ plants. Whole-genome sequencing (HiSeqTM 2500 platform; Illumina, San Diego, CA) of two bulked DNA samples was performed to generate the sequencing library of 200-bp paired-end reads.

BSA ANALYSIS AND PRIMARY MAPPING. The total resequenced reads were analyzed by removing the low-quality reads, 
adaptors, and reads with $>10 \%$ unknown bases. The clean end reads were used across the watermelon reference genome (97103) available in the Cucurbit Genomics Database (Guo et al., 2012, 2019; Zheng et al., 2018), using the Burrows-Wheeler Aligner (BWA) software package (Li and Durbin, 2009). The raw data reads of single-nucleotide polymorphisms (SNPs) and insertion and deletions (InDels) were sorted and low-quality reads ( $<20$ read depth) were removed using Samtool's rmdup command (Li et al., 2009). The Unified Genotyper module of GATK was used to detect the SNPs in multiple samples (McKenna et al., 2010), and the InDels were filtered with variant filtration and annotated with ANNOVAR (Wang et al., 2010). The primary genetic region of the $C l d w$ gene controlling dwarfism was determined by the delta $(\Delta)$ SNP index derived from locally estimated scatterplot smoothing (LOESS) regression $(P \leq 0.01)$ curves at each SNP position of both bulks (dwarf-type and standard-type) according to the following previously reported equations ( $\mathrm{Li}$ et al., 2017):

$$
\begin{gathered}
\text { SNP index }(\mathrm{aa})=(\mathrm{Xaa}+\mathrm{Maa}) \\
\text { SNP index }(\mathrm{bb})=\mathrm{Xaa} /(\mathrm{Xbb}+\mathrm{Mbb}) \\
\Delta(\mathrm{SNP} \text { index })=\mathrm{SNP} \text { index }(\mathrm{aa})-\mathrm{SNP} \text { index }
\end{gathered}
$$

where $\mathrm{X}$ represents the cultivar 812 , M represents the cultivar W1-1, and aa and bb are the dwarf-type bulk and standard type bulk genotypes, respectively. Xaa and Maa indicate the depth of the aa population resulted from $\mathrm{X}$ and $\mathrm{M}$, respectively. $\mathrm{Xbb}$ and $\mathrm{Mbb}$ represent the depth of the bb population resulting from $\mathrm{X}$ and $\mathrm{M}$, respectively. The $\Delta(\mathrm{SNP}$ index $)$ of 1 showed striking correlations between SNPs and dwarf traits, whereas $\Delta$ (SNP in$\operatorname{dex})=0$ represented no association of the SNPs within the dwarf trait. Additionally, the $\Delta$ (SNP index) analysis of each chromosome was conducted for both types of bulk based on the read depth to test the significance of the SNPs at $P \leq 0.01$ and LOESS regression (0.62). The detected region above the threshold value was designated as the main region responsible for controlling dwarfism.

CAPS MARKERS DEVELOPMENT. Initially, the genetic region controlling dwarfism was mapped on targeted chromosome by BSA. All the filtered resequenced data were loaded into SNP2CAPS software (Thiel et al., 2004), and the major SNP loci and differential cutting sites of restriction endonucleases were chosen and transformed into CAPS markers by designing the polymerase chain reaction (PCR) assays across the wholegenome chromosomes (Amanullah et al., 2020). The best CAPS markers for PCR amplification were exported using software (Primer Premier V6.0; Premier Biosoft, San Francisco, CA), and the final sequences were synthesized by Sangon Biotech Co. (Shanghai, China).

CAPS MARKer Verification uSing PCR. The PCR amplification was performed in a $20-\mu \mathrm{L}$ mixture using the touchdown PCR system (Amanullah et al., 2018). The mixture contained 2 $\mu \mathrm{L}$ of template of gDNA, $0.4 \mu \mathrm{L}$ Taq endonuclease, $1 \mu \mathrm{L}$ each of forward and reverse primer, $2 \mu \mathrm{L}$ Taq buffer, $0.6 \mu \mathrm{L}$ dNTPs, and $13 \mu \mathrm{L}$ nuclease-free water. Amplification was started by preheating at $94^{\circ} \mathrm{C}$ for $7 \mathrm{~min}$, followed by 30 heating cycles at $94{ }^{\circ} \mathrm{C}$ for $1 \mathrm{~min}$, denaturation at $60^{\circ} \mathrm{C}$ for $30 \mathrm{~s}$, cooling at $0.5^{\circ} \mathrm{C}$ gradients, extension at $72^{\circ} \mathrm{C}$ for $90 \mathrm{~s}$, and final elongation at $72{ }^{\circ} \mathrm{C}$ for $10 \mathrm{~min}$. All the fragmented PCR products were subsequently separated using $1 \%$ agarose gel electrophoresis and digested with four different restriction enzymes (TaqI, XhoI, EcoRI, and RsaI). The enzyme digestion reaction contained 5 $\mu \mathrm{L}$ of PCR-yielded product, $0.3 \mu \mathrm{L}$ of restriction enzymes, 1 $\mu \mathrm{L}$ of enzyme buffer, and $8.7 \mu \mathrm{L}$ of nuclease-free water. A total of $15 \mu \mathrm{L}$ of digestion reaction was incubated at a specific temperature of 37 or $65^{\circ} \mathrm{C}$, depending on the required temperature for endonuclease activity, in an incubator for 3 to $4 \mathrm{~h}$. The final digested products were verified by the detection of distinct bands in both watermelon cultivars and offspring populations.

Fine MAPPING. The KASP system and PCR reaction procedures were conducted as described previously (Zhao et al., 2017). First, primary linkage mapping was performed on chromosome 9 by using KASP markers genotyping within $184 \mathrm{~F}_{2}$ individuals (137 standard type and 47 dwarf type). Linkage mapping software (JoinMap; Kyazma, Wageningen, the Netherlands) was used by incorporating the data of standard-type (recorded as D) and dwarf-type (recorded as B) $\mathrm{F}_{2}$ individuals. The second-year data of $230 \mathrm{~F}_{2}$ individuals (all dwarf phenotype) were verified for primary mapping by using seven polymorphic CAPS markers (Table 1). Next, two identified major CAPS markers flanking the $C l d w$ gene region were used to identify the recombinant individuals from a total of $390 \mathrm{~F}_{2}$ individuals (all dwarf phenotypes). Furthermore, new KASP markers (Supplemental Table 1) were designed between the detected primary

\begin{tabular}{|c|c|c|c|}
\hline CAPS marker & Primer sequence $\left(5^{\prime} \rightarrow 3^{\prime}\right)$ & Enzyme & Annealing temp $\left({ }^{\circ} \mathrm{C}\right)$ \\
\hline \multirow[t]{2}{*}{$\overline{C 1194526}$} & F: GCTAAATCATCACTTGTTTGCGTAC & TaqI & 55 \\
\hline & R: CTTATGGTATATCTGGTAGCCTTCC & & \\
\hline \multirow[t]{2}{*}{ C1273941 } & F: CCGTCCTCAAGAGAAGTTACCT & XhoI & 55 \\
\hline & R: ACAAGAAGTCGCTCTATGGTCT & & \\
\hline \multirow[t]{2}{*}{ C1466958 } & F: ACGGATGTCCTACTGATCTAAGT & TaqI & 55 \\
\hline & R: CGGCTTCAATGACGCTGTT & & \\
\hline \multirow[t]{2}{*}{ C1652670 } & F: ATGATTGCTGTGGTTGGAATGT & TaqI & 55 \\
\hline & R: CCCTATGTTTCTGCCCTCCC & & \\
\hline \multirow[t]{2}{*}{ C1763379 } & F: CAACATGATCCGACACGAACC & TaqI & 55 \\
\hline & R: TTGTCAATGGAATGATGCACCA & & \\
\hline \multirow[t]{2}{*}{ C1906994 } & F: CTCAGCATAACCAGTCAGTAGTATA & EcoRI & 55 \\
\hline & R: GTGGACGGCAATGATAGCAA & & \\
\hline \multirow[t]{2}{*}{$C 2415514$} & F: GAATACGGAGGACACCATAGTTG & RsaI & 55 \\
\hline & R: AGAACATAAGAACCAGAGACGATAC & & \\
\hline
\end{tabular}

Table 1. Primer sequences of the cleaved amplified polymorphism sequences (CAPS) markers used to map the Cldw gene controlling compact and dwarf growth habit in watermelon. 
mapping interval and further validated by using the 43 identified recombinant individuals.

Exogenous GA 3 TREATMEnT, ENdogenous GA 3 MEASUREMENTS, AND RELATIVE EXPRESSION ANALYSIS. Initially, the two cultivars W1-1 and 812 were exogenously treated with $\mathrm{GA}_{3}$ in a large plastic greenhouse. The $\mathrm{GA}_{3}$ used for exogenous treatment was obtained from Solarbio Science and Technology (Beijing, China). The $\mathrm{GA}_{3}$ powder was dissolved in a small quantity of ethanol and diluted in double-distilled water $\left(\mathrm{ddH}_{2} \mathrm{O}\right)$ to make the final solution. All plants were exogenously treated with various $\mathrm{GA}_{3}$ concentrations $\left(0.3,0.9\right.$, and $\left.1.5 \mathrm{mmol} \cdot \mathrm{L}^{-1}\right)$; control plants were simply sprayed with a mixture of ethanol and $\mathrm{ddH}_{2} \mathrm{O}$. The vine length was measured once per week after the seed germination.

When the plants reached the same visual growth rate as that during their reproductive growth stage, the eighth internodes from three plants were sampled. The endogenous hormone contents of sampled internodes were extracted by using liquid chromatography-mass spectrometry according to a previously described method (Chen et al., 2011). The actual measurement of endogenous $\mathrm{GA}_{3}$ was performed for the same harvested node samples.

The gene expression level was analyzed using a quantitative real-time (qRT) PCR system (QTOWER; Analytik Jena, Jena, Germany) with the SYBR Green Master Mix reagent (Novogene, Beijing, China) according to the manufacturer's instructions. The qRT-PCR reaction mixture was composed of $1 \mu \mathrm{L}$ of cDNA (50 ng. $\mu \mathrm{L}^{-1}$ of total RNA), $1 \mu \mathrm{L}$ of each primer (10 $\mu \mathrm{M}), 10 \mu \mathrm{L}$ of $2 \times$ SYBR Green Master Mix, and nuclease-free water, with a final volume of $20 \mu \mathrm{L}$. The amplification reactions included 1 cycle of $95^{\circ} \mathrm{C}$ for $1 \mathrm{~min}$, followed by 35 amplification cycles at $95^{\circ} \mathrm{C}$ for $15 \mathrm{~s}, 58^{\circ} \mathrm{C}$ for $20 \mathrm{~s}$, and $72^{\circ} \mathrm{C}$ for $15 \mathrm{~s}$. The specific transcript amplification was verified by a single peak of the melting curve analysis obtained after the completion of the amplification reaction. The negative controls without cDNA templates were performed for overall runs to verify the potential impurity.

RNA EXTRACTION AND CLONING OF $\boldsymbol{C L A 0 1 5 4 0 7 . ~ P l a n t s ~ a d j a - ~}$ cent to the eighth internode during the reproductive growth period (ovary expansion stage) were independently harvested from both watermelon cultivars for the tissue-specific analysis. Total RNA from the harvested samples was isolated using Trizol (Zhang et al., 2006). Complementary DNA (cDNA) was synthesized using a ReverTra Ace qPCR RT Kit (Toyobo, Osaka, Japan).
Cloning of the candidate gene Cla015407 sequence was performed for both cultivars. Amplification was performed as follows: 1 cycle of $95^{\circ} \mathrm{C}$ for $5 \mathrm{~min}$, followed by 30 amplification cycles at $95^{\circ} \mathrm{C}$ for $30 \mathrm{~s}, 50^{\circ} \mathrm{C}$ for $30 \mathrm{~s}$, and $72^{\circ} \mathrm{C}$ for $90 \mathrm{~s}$. Then, the amplified targeted fragments were aligned to the vector pMD18-T and sent to Sangon Biotech Co. for sequencing.

Data ANalysis. All the numerical data of plant phenotypes were entered and preliminarily sorted using spreadsheet software (Excel; Microsoft, Redmond, WA). The standard descriptive analysis of means and genetic segregation analysis $\left(\chi^{2}\right.$ test $)$ were performed using statistical analysis software (SPSS 23.0; IBM, Armonk, NY).

\section{Results}

ANALYSIS OF PHENOTYPES AND GENETIC INHERITANCE OF WATERMELON DWARFISM. The vine length of the cultivars W1-1 and 812 clearly exhibited significant differences in dwarfism at multiple growth points, standard stem lengths, and fewer growth points during the entire growth period, respectively (Fig. 1). The average stem lengths of cultivars 812 and W1-1 were $53.3 \mathrm{~cm}$ and $171.10 \mathrm{~cm}$ in 2018 , and the stem lengths were $56.6 \mathrm{~cm}$ and $173.90 \mathrm{~cm}$ in 2019 , respectively. Overall, the stem length did not show any type of changes in the $\mathrm{F}_{2}$-derived individuals; the maximum stem length was $186.3 \mathrm{~cm}$ and the minimum stem length was $40.7 \mathrm{~cm}$.

The results of the analysis of the genetic segregation ratio of the population of six generations are shown in Table 2. In 2018, the expressed the ratio of standard $(n=149)$ to dwarf $(n=47)$ types of $196 \mathrm{~F}_{2}$-derived progenies corresponded to a 3:1 Mendelian segregation ratio $\left(\chi^{2}=0.1429 ; P>0.05\right)$. In 2019, a total of 1096 standard-type and 343 dwarf-type $F_{2}$ plants were perfectly segregated among $1439 \mathrm{~F}_{2}$-derived individuals, which also supported the previously observed phenotypic segregation ratio of $3: 1\left(\chi^{2}=1.0718 ; P>0.05\right)$. The plants of both $\mathrm{F}_{1}$ and $\mathrm{BC}_{1} \mathrm{P} 1$ populations behaved as the standard type; however, the plants of the $\mathrm{BC}_{1} \mathrm{P} 2$ population exhibited a 1:1 segregation ratio of the standard type $(n=73)$ and dwarf type $(n=77)$. Overall, the observed segregation ratios of the standard-type and dwarf-type phenotypes in all derived generations indicated that the internode length is primarily controlled by a single recessive gene, $C l d w$; however, the standard-type internode length was dominant over that of the dwarf-type, and the genetic inheritance of the observed individuals was unaffected in the environment during 2018 and 2019.

Table 2. Verification of $\mathrm{P} 1, \mathrm{P} 2, \mathrm{~F}_{1}, \mathrm{~F}_{2}, \mathrm{BC}_{1} \mathrm{P} 1$, and $\mathrm{BC}_{1} \mathrm{P} 2$ segregation ratios of the standard-type and dwarf-type watermelon individuals.

\begin{tabular}{|c|c|c|c|c|c|c|}
\hline Yr & Group & Standard type (no.) & Dwarf type (no.) & Expected $^{\mathrm{z}}$ ratio & Actual $^{\mathrm{y}}$ & $x^{2 x}$ \\
\hline 2018 & P1 & 15 & 0 & $-^{\mathrm{w}}$ & - & - \\
\hline 2018 & $\mathrm{P} 2$ & 0 & 15 & - & - & - \\
\hline 2018 & $\mathrm{~F}_{1}$ & 15 & 0 & 一 & - & - \\
\hline 2018 & $\mathrm{BC}_{1} \mathrm{P} 2$ & 73 & 77 & $1: 1$ & $0.95: 1$ & 0.1133 \\
\hline 2018 & $\mathrm{~F}_{2}$ & 149 & 47 & $3: 1$ & $3.17: 1$ & 0.1429 \\
\hline 2019 & $\mathrm{~F}_{2}$ & 1096 & 343 & $3: 1$ & $3.20: 1$ & 1.0718 \\
\hline
\end{tabular}

${ }^{\mathrm{z}}$ Expected standard type:dwarf type ratio.

${ }^{y}$ Actual standard type:dwarf type ratio.

${ }^{\mathrm{x}}$ The standard fixed value of $\chi_{0.05}^{2}=3.84 ; \chi^{2}<3.84$ showed no significance in the differences.

${ }^{\mathrm{w}}$ Invalid value. 
IdentifiCATION OF THE $C_{L D W}$ GENE USING BSA AND PRIMARY MAPPING. The results of whole-genome sequencing and BSA of two constructed DNA bulks (30 dwarf types and 30 standard types) revealed the primary genetic region of the $C l d w$ gene on chromosomes 9 with a fluctuating peak (Fig. 2, Supplemental Table 2). However, the main genetic region of $C l d w$ on chromosome 9 ranged from 1.20 to $3.57 \mathrm{Mb}$ (Fig. 3). A total of 10 KASP markers were developed in the candidate gene region, which was detected by the BSA analysis; primary linkage mapping was performed according to the observed phenotypic data of $184 \mathrm{~F}_{2}$-derived individuals (137 standard types and 47 dwarf types). The main genetic locus of the dwarfism controlling gene was spotted between the adjacent KASP markers K1692577 and K1906994 within a 4.8-cM interval (Fig. 4), and the physical distance was $214.4 \mathrm{~kb}$ (range, 1,692,577-1,906,994 bp).

In 2019, additional primary mapping was performed by further developing seven CAPS markers at the chromosome 9 region to screen the $230 \mathrm{~F}_{2}$ individuals with the dwarf phenotype. Furthermore, the Cldw gene was delimited between two CAPS markers, $C 1652670$ and C1906994, from three and five recombinants $(\mathrm{H})$, respectively (Fig. 4); however, a single marker, C1763379, seemed perfectly co-segregated by exhibiting the dwarf-type (B) trait. The CAPS markers showed more recombinants, but there were no recombinants in marker C1763379. The results of primary mapping were similar for watermelon grown during the experiments performed in 2018 and 2019, which indicated the perfect mapping interval.

Fine MAPPING. Initially, a large group of $390 \mathrm{~F}_{2}$ individuals (all dwarf-types) was used for screening and verification of two CAPS markers, C1652670 and C1906994, and a total of 43

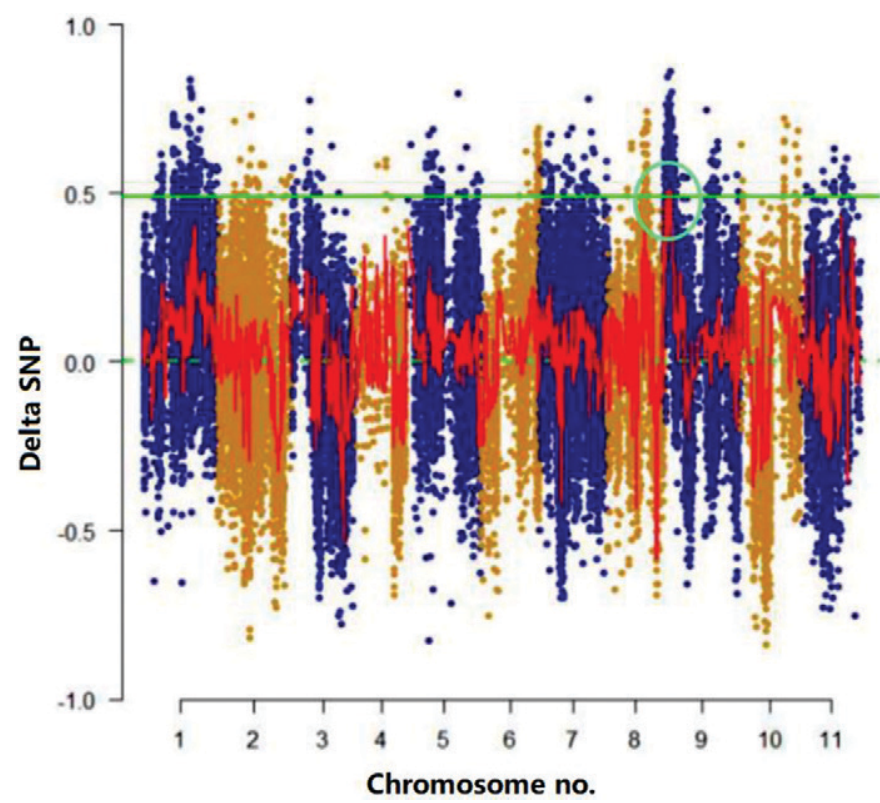

Fig. 2. Delta single-nucleotide polymorphism (SNP) index of the whole-genome chromosomes to identify the compact growth habit gene locus in watermelon. The scattered points indicate the delta SNP index calculated for each specific SNP position. The $\mathrm{x}$-axis represents the position of the watermelon chromosomes; the y-axis represents the SNP index. The curved red lines on the $\mathrm{x}$-axis represent an average of each chromosome and indicate the SNP index. The green line is the threshold level determined using locally estimated scatterplot smoothing (LOESS) regression. The green circle on chromosome 9 represents the significant fluctuation peak detected in the main genetic region.

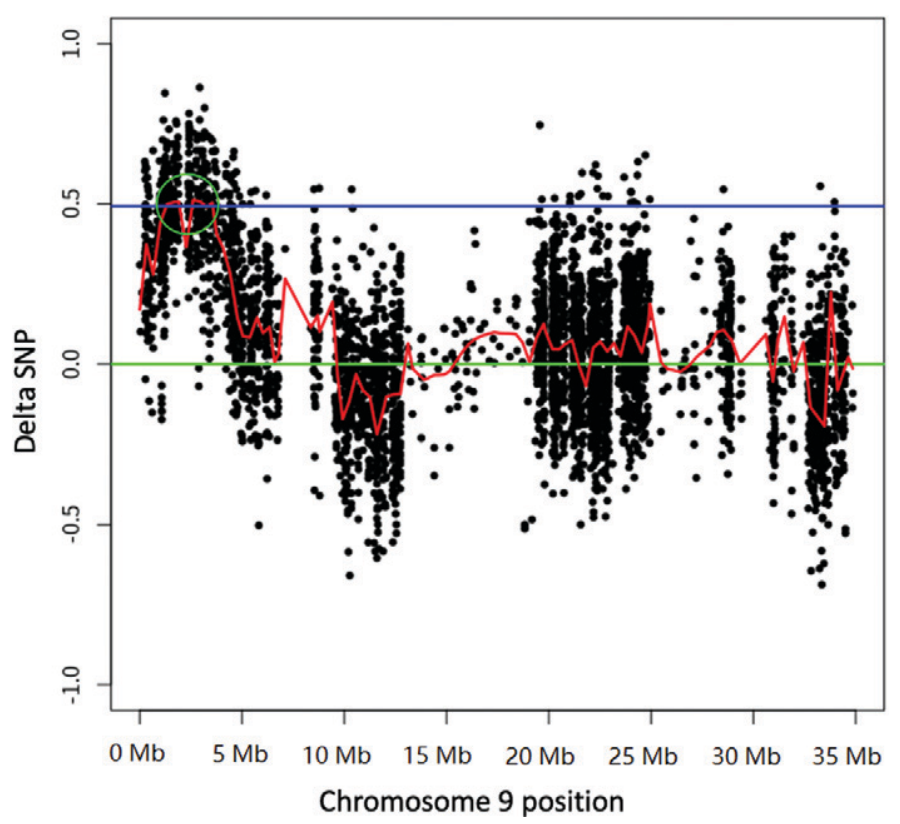

Fig. 3. Delta single-nucleotide polymorphism (SNP) index plots across chromosome 9 to identify the compact growth habit gene locus in watermelon. The $\mathrm{x}$-axis represents the position of watermelon chromosome 9; the y-axis represents the SNP index. The curved red lines on the $\mathrm{x}$-axis represent the average of each chromosome and indicate the SNP index. The candidate gene region is indicated by a green circle and ranges from 1.20 to $3.57 \mathrm{Mb}$. The blue line is the threshold level $(0.50)$ determined by locally estimated scatterplot smoothing (LOESS) regression (0.62) on chromosome 9.

recombinants were mainly filtered from primary mapping analysis. Then, 5 KASP markers, K1692577, K1764972, K1822206, $K 1865395$, and $K 1906994$, were further developed and used for fine mapping using a genotypic analysis of 43 recessive recombinants (Wang et al., 2019). Finally, the Cldw gene was mapped between two KASP markers, K1822206 and K1865395 (Fig. 5), at the delimited region of $43.2 \mathrm{~kb}$.

According to the annotated online version of the reference genome of watermelon, only two homologous genes, Cla015407 and Cla015408, were found in fine-mapped regions between 1,822,206 and 1,865,395 bp, and these two genes were predicted to encode GA $3 \beta$-hydroxylase protein, which mainly triggers the catalytic activity of conversion of nonbioactive GA into bioactive GA (Teng et al., 2013). Bioactive GA promotes elongation in the vine length, but the dwarfism is caused by the absence of bioactive GA. Many studies reported that a gene mutation of $\mathrm{GA}_{3}$ hydroxylase causes the plant compact and dwarf growth habit (Itoh et al., 2001). In this study, various exogenous $\mathrm{GA}_{3}$ treatments were applied to identify the relationship of dwarfism and GA in watermelon cultivar 812. The endogenous $\mathrm{GA}_{3}$ quantification (nanograms per gram) indicated that $\mathrm{GA}_{3}$ deficiency is the only factor that causes dwarfism in cultivar 812 .

Furthermore, a KASP marker was successfully developed to identify the candidate gene for controlling dwarfism by using the detected SNP mutations in gene Cla015408. Then, this KASP marker, KCla015408, was genotyped in 43 recombinants, but genetically recombinant individuals were found. Therefore, the gene Cla015408 was ignored and the gene Cla015407 was specified as a candidate gene for controlling dwarfism in cultivar 812 . 
(A)

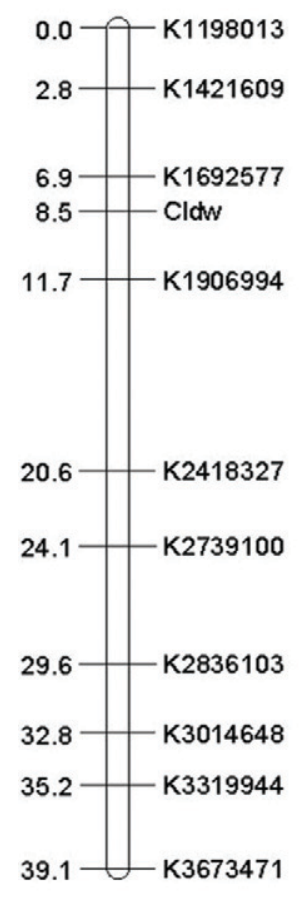

(B)

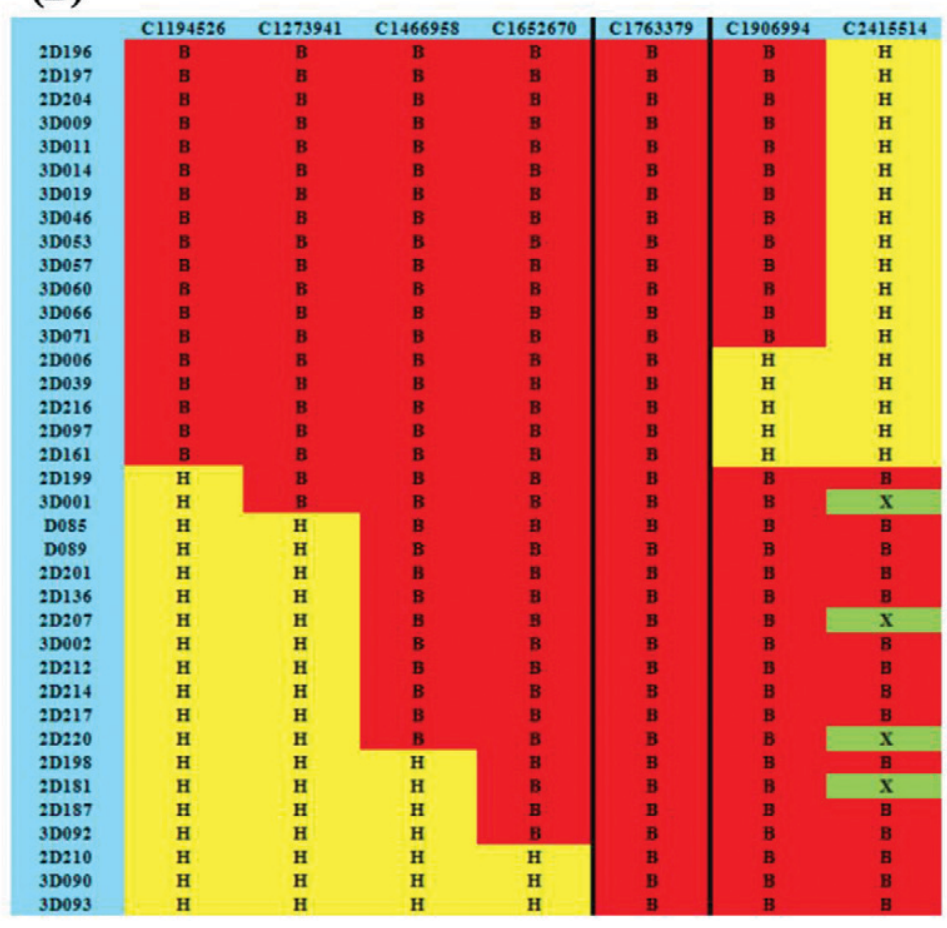

Fig. 4. Primary mapping of compact growth habit gene $C l d w$ in watermelon using (A) kompetitive allele-specific polymerase chain reaction (KASP) and (B) cleaved amplified polymorphism sequence (CAPS) marker genotyping. (A) Linkage map of $10 \mathrm{KASP}$ markers in the candidate region. The entire map distance was $39.10 \mathrm{cM}$, and the average distance was 3.91 cM. The $C l d w$ gene was preliminarily mapped between markers K1692577 and K1906994 on chromosome 9 using a total of $184 \mathrm{~F}_{2}$ individuals (137 standard type and 47 dwarf type). The KASP markers are on the right side and the genetic interval for each marker is on the left side. (B) The $C l d w$ gene was further mapped at marker $C 1763379$ between two markers $C 1652670$ and $C 1906994$ on chromosome 9 using a total of $230 \mathrm{~F}_{2}$ dwarf-type individuals. Marker genotypes of the recombinants near the dwarf gene $C l d w$ were between markers $C 1652670$ and $C 1906994$. The alleles are abbreviated according to their origin: $\mathrm{B}$ represents the dwarf-type, $\mathrm{H}$ represents the dwarf-type recombinants, and $\mathrm{X}$ represents the missing date. The blue column shows $\mathrm{F}_{2}$ individuals. The blue row shows CAPS markers.

EXPRESSION AND CLONING OF DWARFISM GENE $C_{L A} 015407$ AND GA $_{3}$ MEASUREMENT. To further explore whether the Cla015407 gene sequence and structure are mutated, we amplified the fulllength gene sequence (including the intron sequence) in two cultivars, W1-1 and 812. According to the comparative analysis of the Cla015407 gene sequence, watermelon cultivar sequences, and the published watermelon reference genome (97103), the structure of the Cla015407 gene contains two exons and one intron. The coding sequence (including intron sequence) of the Cla015407 gene showed two single base mutations and a single base insertion in cultivar 812; however, this coding sequence had a single base mutation that occurred at the splice site of the intron in cultivar 812. The three SNP mutation sites were located in the intron region of the Cla015407 gene (Fig. 6C).

In general, single-base mutations and insertions or deletions in introns did not affect gene expression. However, regarding the three mutations detected, one site in the Cla015407 gene was changed from $\mathrm{G}$ to $\mathrm{A}(\mathrm{G} \rightarrow \mathrm{A})$ in cultivar 812 , and the main mutation site was located at the splice site region of the intron of the Cla015407 gene (Fig. 6C). Interestingly, the original splicing site of the Cla015407 gene in cultivar 812 mutated from AG to AA, and the new splicing site $\mathrm{AG}$ appeared to alter the original exon length during the transcription process. Therefore, we speculated that this new altered splicing site allowed normal expression of the affected gene. Therefore, the splice site region mutation in the Cla015407 gene in cultivar 812 was mainly caused by gene structure mutation that affected the normal gene expression and exhibited dwarfism in cultivar 812 .

If the watermelon plants exhibit the same dwarfism mechanism, then they must be deficient in bioactive GA. For further confirmation of whether this dwarfism is related to bioactive GA, we measured endogenous $\mathrm{GA}_{3}$ synthesis at the eighth internode of cultivars W1-1 and 812; the results indicated that more bioactive $\mathrm{GA}_{3}$ was synthesized in cultivar W1-1 than in cultivar 812 (Fig. 6A). The expression level of the Cla015407 candidate gene in both cultivars was also analyzed by qRT-PCR; the results revealed a significantly higher expression level in W1-1 than in 812 (Fig. 6B). However, the expression level of the Cla015407 gene and $\mathrm{GA}_{3}$ contents indicated improved synthesis of bioactive $\mathrm{GA}_{3}$ in cultivar W1-1 and that the gene expression level was also positively correlated with the endogenous $\mathrm{GA}_{3}$ contents.

Exogenous GA $_{3}$ TREATMENT. If the Cla015407 gene controls dwarfism, then dwarfism should be induced by gene mutation and endogenous bioactivity of $\mathrm{GA}_{3}$. Therefore, we hypothesized that exogenous treatment of $\mathrm{GA}_{3}$ can exhibit phenotypic differences in both cultivars. Three distinct $\mathrm{GA}_{3}$ treatments $(0.3,0.9$, and 1.5 $\mathrm{mmol} \cdot \mathrm{L}^{-1}$ ) were exogenously applied on plants of both cultivars. Cultivar 812 showed a significant response to increased $\mathrm{GA}_{3}$ exogenous treatment. No significant difference in the vine length of both cultivars was induced by the maximum $\mathrm{GA}_{3}$ concentration $\left(1.5 \mathrm{mmol} \cdot \mathrm{L}^{-1}\right)$. The treatment was continued until the plants reached the reproductive growth stage. Cultivars W1-1 and 812 exhibited no significant differences in vine length in response to the $\mathrm{GA}_{3}$ treatment $\left(1.5 \mathrm{mmol} \cdot \mathrm{L}^{-1}\right)$; however, the number of side branches was significantly decreased in response to the maximum $\mathrm{GA}_{3}$ concentration (Fig. 7). These results strongly indicated that the vine length differences of cultivars W1-1 and 812 occurred because of the differences in the $\mathrm{GA}_{3}$ concentration.

\section{Discussion}

The compact and dwarf growth habit is one of the most important phenotypes of watermelon plants, and dwarf-type plants 


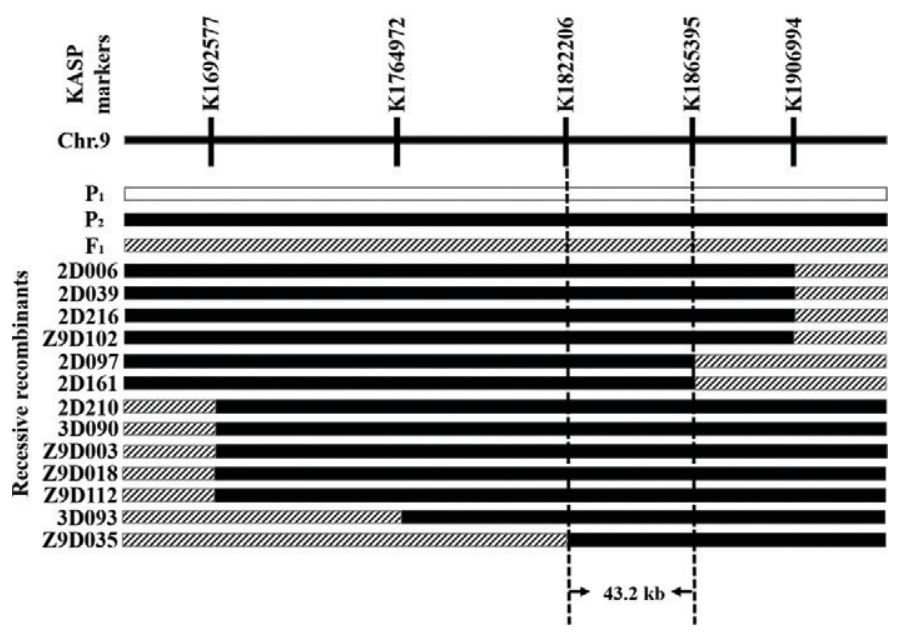

Fig. 5. Fine mapping of the $C l d w$ gene controlling the compact and dwarf growth habit in watermelon through recessive recombinants. The gene $C l d w$ was delimited in the $43.2 \mathrm{~kb}$ region of chromosome 9 between kompetitive allele-specific polymerase chain reaction (KASP) markers K1822206 and K1665395. The white shows bar W1-1 (P1), the black bar shows 812 (P2), the gray bar shows $\mathrm{F}_{1}$, and 2D006, 2D039, 2D216, Z9D102, 2D097, 2D161, 2D210, 3D090, Z9D003, Z9D018, Z9D112, 3D093, and Z9D035 are dwarf-type recessive recombinants.

are suitable for high-density cultivation because of their compact nature (Dong et al., 2018). From the farmer's point of view, dwarf plants use more natural light, cover less land area, save

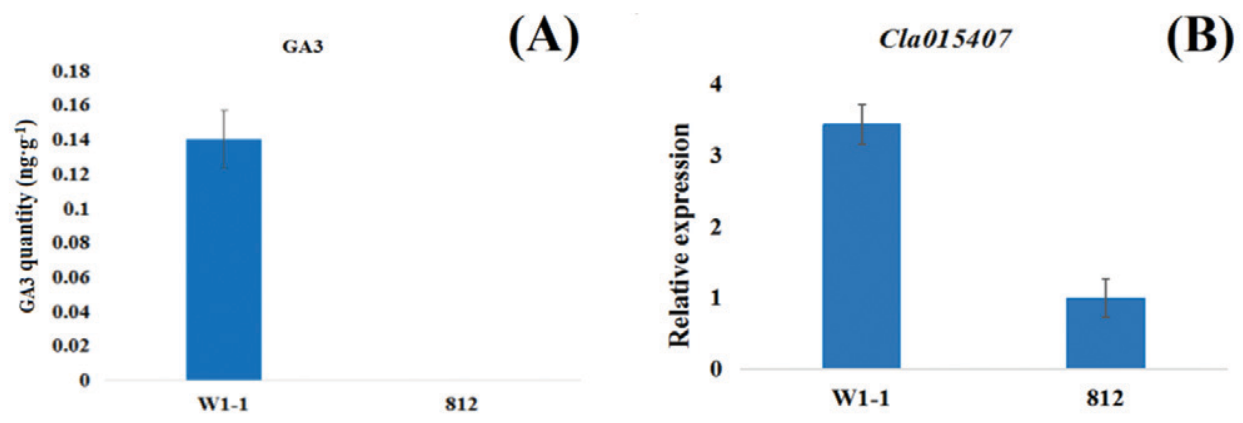

(C)

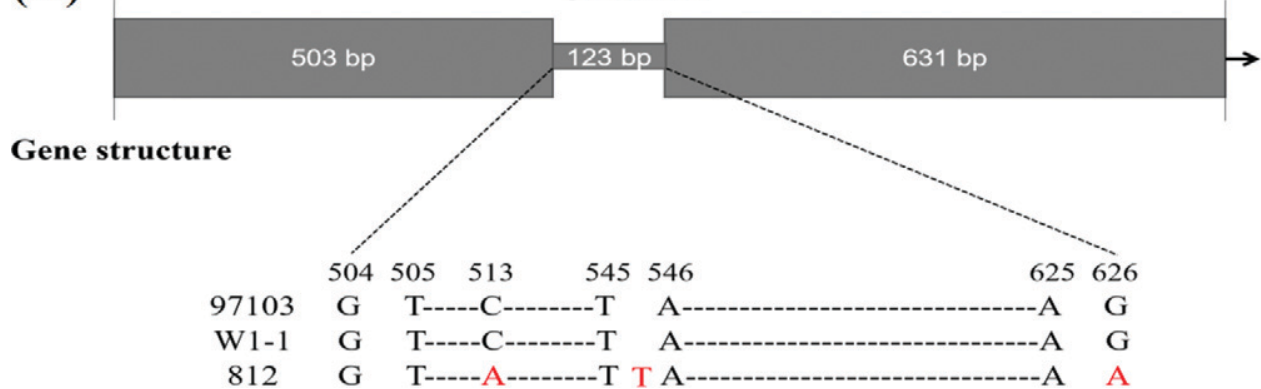

Fig. 6. Validation of the $C l d w$ gene that controls the compact and dwarf growth habit of watermelon. (A) Measurement of the endogenous gibberellic acid $\left(\mathrm{GA}_{3}\right)$ content (nanograms per gram) in the eighth internodes during the reproductive growth period of two cultivars, W1-1 (P1; standard type) and 812 (P2; dwarf-type). Error bar indicates the SD of three repeats $(n=3)$. Values are means \pm SD $(n=3)$. (B) The expression levels of the Cla015407 gene in the eighth internodes during the reproductive growth period of two cultivars, P1 and P2. The expression level of the Cla015407 gene in P2 was set to a value of 1 and used as a reference. Error bar indicates the SD of three repeats $(\mathrm{n}=3)$. Values are means $\pm \mathrm{SD}(\mathrm{n}=3)$. (C) Comparative analysis of genomic variations of the Cla010407 gene between cultivars and the reference watermelon genome (97103). The large gray boxes indicate the exon and the small gray boxes indicate the intron. The red letters indicate mutations and insertion of bases. Two single-base mutations and one single-base insertion in the intron were detected for P2. One of the singlebase mutations occurred at the splice site of the intron. labor costs, need no pruning, have reduced production costs, and result in increased profits, among other benefits. Therefore, the selection of excellent cultivars of dwarf plants should be considred for the important selection standard of production of waterars are cultivated less frequently because of improper availabilion cultivars for further commercial cultivation. (Yamaguchi, 2008), and it is reason why the no synthesis of bioactive GA (Itoh et al., 2001; Teng et al., Teng et al., 2013).

During this study, a fine mapping analysis indicated that the $C l d w$ region is located on chromosome 9, and that two main genes $\mathrm{Cla015407}$ and $\mathrm{ClaO} 15408$ are directly involved in the synthesis of bioactive GA. Two experiments were conducted to verify whether the dwarfism of cultivars W1-1 and 812 is related to bioactive GA. Initially, exogenous $\mathrm{GA}_{3}$ treatment at a maximum concentration of 1.5 $\mathrm{mmol} \cdot \mathrm{L}^{-1}$ at the plant reproductive growth stage demonstrated that the vine length of cultivar 812 retained the wild-type phenotype. This result also indicated that the dwarf-type cultivar 812 has hormonal mutations because of gene dysfunction in the GA synthesis pathway. Subsequently, the endogenous $\mathrm{GA}_{3}$ content of cultivar 812 was significantly decreased at the eighth internode compared with that of W1-1, which strongly indicated that $\mathrm{GA}_{3}$ was not synthesized properly in 812. These two conclusions also indicated that plant dwarfism is caused by a loss of function or abnormal function of homologous genes Cla015407 and Cla015408.

We developed a KASP marker based on the SNP positions of the Cla015408 gene to precisely identify these two homologous genes as targeted genes of dwarfism. The Cla015408 gene was not effective because of the co-segregation of genetic recombination in 43 recombinants. Therefore, the Cla015408 gene was excluded and the Cla015407 gene was considered the final candidate gene. Then, gene expression analyses 


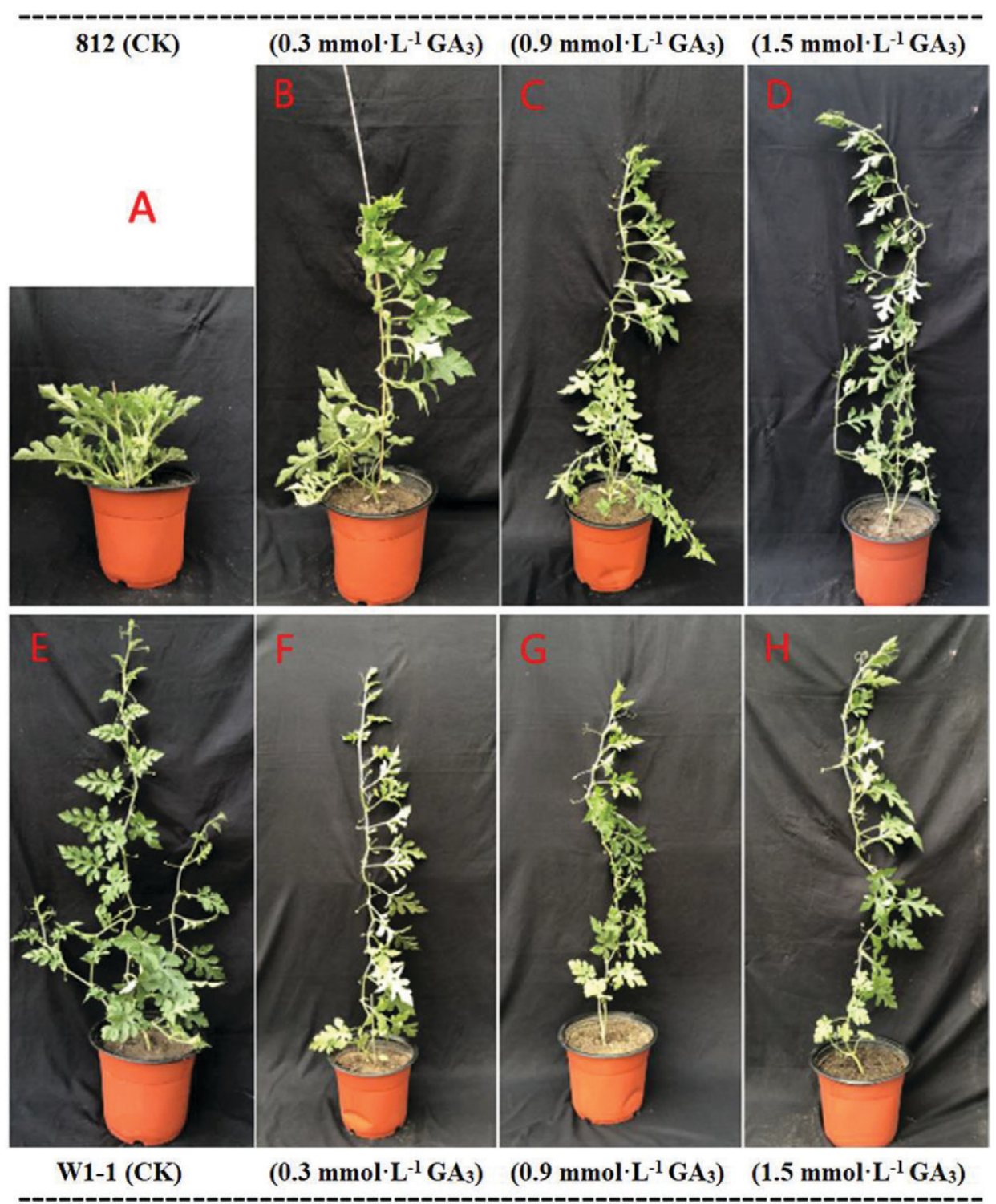

Fig. 7. The differences in plant morphological characteristics (height and internodal length) of watermelon cultivars W1-1 (P1; standard type) and 812 (P2; dwarf type) after various concentrations of gibberellic acid (GA $)$ treatment. (A-D) Control check (CK) treatment and various exogenous $\mathrm{GA}_{3}$ treatments for P2. (E-H) CK treatment and various exogenous $\mathrm{GA}_{3}$ treatments for P1. Control plants were treated with a simple spray of an equal volume of a mixture of ethanol and double-distilled water. Photographs were obtained when the plants reached the reproductive growth period.

were conducted using qRT-PCR for cultivars W1-1 and 812, and the results demonstrated that the expression of the Cla015407 gene was significantly greater in cultivar W1-1 than in 812, and strongly indicated that the quantity of bioactive GA synthesis was greater in cultivar W1-1.

During this study, the GA $3 \beta$-hydroxylase gene was found in the fine-mapped region of $43.2 \mathrm{~kb}$. The comparative analysis of the Cla015407 gene coding sequence, sequencing data of both cultivars W1-1 and 812, and the reference genome of watermelon (97103) exhibited a total of three SNP mutation sites in the intron region and a splice site mutation was found in the Cla015407 gene sequence. According to the $\mathrm{GT} \rightarrow \mathrm{AG}$ rule $(\mathrm{Xu}$ et al., 1995), the two nucleotides starting at the $5^{\prime}$ end of any intron were GT, but the two nucleotides present at the $3^{\prime}$ end were AG. Interestingly, the original splice site region of the
Cla015407 gene in cultivar 812 mutated from AG to AA and appeared as a new AG splicing site. In summary, all evidence presented herein strongly suggest that the GA $3 \beta$-hydroxylase homolog of Cla015407 might be a causal gene that controls dwarfism in watermelon cultivar 812 .

\section{Literature Cited}

Allen, G.C., M.A. Flores-Vergara, S. Krasynanski, S. Kumar, and W.F. Thompson. 2006. A modified protocol for rapid DNA isolation from plant tissues using cetyltrimethylammonium bromide. Nat. Protoc. 1:2320-2325, doi: 10.1038/nprot. 2006.384.

Amanullah, S., A. Saroj, B.A. Osae, S. Liu, H.Y. Liu, P. Gao, and F.S. Luan. 2020. Detection of putative QTL regions associated with ovary traits in melon using SNP-CAPS markers. Scientia Hort. 270:109445, doi: 10.1016/j. scienta.2020.109445.

Amanullah, S., S. Liu, P. Gao, Z.C. Zhu, Q.L. Zhu, C. Fan, and F.S. Luan. 2018. QTL mapping for melon (Cucumis melo L.) fruit traits by assembling and utilization of novel SNPs based CAPs markers. Scientia Hort. 236:18-29, doi: 10.1016/j.scienta.2018.02.041.

Amasino, R.M., F.M. Schomburg, S.D. Michaels, and C.M. Bizzell. 2003. Dwarfism genes and dwarf plants. Wisconsin Alumni Res. Foundation 1:2.

Chen, Y., M.M. Hou, L.J. Liu, S. $\mathrm{Wu}, \mathrm{Y}$. Shen, K. Ishiyama, M. Kobayashi, D.R. McCarty, and B.C. Tian. 2014. The maize dwarf1 encodes a gibberellin 3-oxidase and is dual localized to the nucleus and cytosol. Plant Physiol. 166:2028-2039, doi: 10.1104/pp. 114.247486 .

Chen, M.L., Y.Q. Huang, J.Q. Liu, B.F. Yuan, and Y.Q. Feng. 2011. Highly sensitive profiling assay of acidic plant hormones using a novel massprobe by capillary electrophoresis-time of flight-mass spectrometry. J. Chromatogr. B Analyt. Technol. Biomed. Life Sci. 879:938-944, doi: 10.1016/j.jchromb.2011.03.003.

Chiang, H.H., I. Hwang, and H.M. Goodman. 1995. Isolation of the Arabidopsis GA4 locus. Plant Cell 7:195-201, doi: 10.2307/3869995.

Dong, W., D. Wu, G. Li, D. Wu, and Z. Wang. 2018. Next-generation sequencing from bulked segregant analysis identifies a compact growth habit gene in watermelon. Sci. Rep. 8:2908, doi: 10.1038/ s41598-018-21293-1.

Dyutin, K.E. and E.A. Afanas'eva. 1987. Inheritance of the short vine trait in watermelon. Cytol. Genet. 21:71-73.

Guo, S., J. Zhang, H. Sun, J. Salse, W.J. Lucas, H. Zhang, Y. Zheng, L. Mao, Y. Ren, Z. Wang, J. Min, X. Guo, F. Murat, B.K. Ham, Z. Zhang, S. Gao, M. Huang, Y. Xu, S. Zhong, A. Bombarely, L.A. Mueller, H. Zhao, H. He, Y. Zhang, Z. Zhang, S. Huang, T. Tan, E. 
Pang, K. Lin, Q. Hu, H. Kuang, P. Ni, B. Wang, J. Liu, Q. Kou, W. Hou, X. Zou, J. Jiang, G. Gong, K. Klee, H. Schoof, Y. Huang, X. Hu, S. Dong, D. Liang, J. Wang, K. Wu, Y. Xia, X. Zhao, Z. Zheng, M. Xing, X. Liang, B. Huang, T. Lv, J. Wang, Y. Yin, H. Yi, R. Li, M. Wu, A. Levi, X. Zhang, J.J. Giovannoni, J. Wang, Y. Li, Z. Fei, and Y. Xu. 2012. The draft genome of watermelon (Citrullus lanatus) and re-sequencing of 20 diverse accessions. Nat. Genet. 45:51-58, doi: $10.1038 /$ ng.2470.

Guo, S., S. Zhao, H. Sun, X. Wang, S. Wu, T. Lin, Y. Ren, L. Gao, Y. Deng, J. Zhang, X. Lu, H. Zhang, J. Shang, G. Gong, C. Wen, N. He, S. Tian, M. Li, J. Liu, Y. Zhu, R. Jarret, A. Levi, X. Zhang, S. Huang, Z. Fei, W. Liu, and Y. Xu. 2019. Resequencing of 414 cultivated and wild watermelon accessions identifies selection for fruit quality traits. Nat. Genet. 51:1616-1623, doi: 10.1038/s41588-0190518-4.

Hedden, P. and A.L. Phillips. 2001. Gibberellin metabolism: New insights revealed by the genes. Trends Plant Sci. 5:523-530, doi: 10 . 1016/S1360-1385(00)01790-8.

Huang, H., X. Zhang, Z. Wei, Q. Li, and Q. Li. 1995. The study of male sterile watermelon with short vine. China Cuc. Veg. 6-9, doi: 10. 16861/j.cnki.zggc.1995.03.004. (In Chinese).

Huang, H., X. Zhang, Z. Wei, Q. Li, and Q. Li. 1998. Inheritance of male-sterility and compact growth habit in watermelon [Citrullus lanatus (Thunb.) Matsum. and Nakai]. Sci. Hort. 74:175-181, doi: 10. 1016/S0304-4238(97)00102-7.

Hwang, J., J. Oh, Z. Kim, J.E. Staub, S.M. Chung, and Y. Park. 2014. Fine genetic mapping of a locus controlling short internode length in melon (Cucumis melo L.). Mol. Breed. 34:949-961, doi: 10.1007/ s11032-014-0088-1.

Itoh, H., M. Ueguchi-Tanaka, N. Sentoku, H. Kitano, M. Matsuo$\mathrm{ka}$, and M. Kobayashi. 2001. Cloning and functional analysis of two gibberellin 3 beta hydroxylase genes that are differently expressed during the growth of rice. Proc. Natl. Acad. Sci. USA 98: 8909-8914, doi: 10.1073/pnas.141239398.

Kauffman, C.S. and R.L. Lower. 1976. Inheritance of an extreme dwarf plant type in the cucumber. J. Amer. Soc. Hort. Sci. 101:150-151.

Knavel, D.E. 1988. Ky-P, short-internode muskmelon. HortScience 23:224.

Knavel, D.E. 1990. Inheritance of a short-internode mutant of 'Mainstream' muskmelon. HortScience 25:1274-1275, doi: 10.1007/ BF00023642.

Kubicki, B., U. Soltysiak, and A. Korzeniewska. 1986. Induced mutation in cucumber (Cucumis sativus L.) V. Compact type of growth. Genet. Pol. 27:289-298.

Li, B., Y.L. Zhao, Q.L. Zhu, Z.P. Zhang, C. Fan, S. Amanullah, P. Gao, and F.S. Luan. 2017. Mapping of powdery mildew resistance genes in melon (Cucumis melo L.) by bulked segregant analysis. Scientia Hort. 220:160-167, doi: 10.1016/j.scienta.2017.04.001.

Li, H., B. Handsaker, A. Wysoker, T. Fennell, J. Ruan, N. Homer, G. Marth, G. Abecasis, and R. Durbin. 2009. The sequence alignment/ map format and SAMtools. Bioinformatics 25:2078-2079, doi: 10. 1093/bioinformatics/btp352.

Li, H. and R. Durbin. 2009. Fast and accurate short read alignment with Burrows-Wheeler transform. Bioinformatics 25:1754-1760, doi: 10.1093/bioinformatics/btp324.

Li, Y., L. Yang, M. Pathak, D. Li, X. He, and Y. Weng. 2011. Fine genetic mapping of $c p$ : A recessive gene for compact (dwarf) plant architecture in cucumber, Cucumis sativus L. Theor. Appl. Genet. 123:973-983, doi: 10.1007/s00122-011-1640-6.

Liu, L., T.T. Sun, X.Y. Liu, Y. Guo, X. Huang, P. Gao, and X.Z. Wang. 2019. Genetic analysis and mapping of a striped rind gene (st3) in melon (Cucumis melo L.). Euphytica 215:20, doi: 10.1007/ s10681-019-2353-1.

Loy, J.B. and P.B.W. Liu. 1972. Inheritance and morphology of two dwarf mutants in watermelon. J. Amer. Soc. Hort. Sci. 97:745-748.

McKenna, A., M. Hanna, E. Banks, A. Sivachenko, K. Cibulskis, A. Kernytsky, K. Garimella, D. Altshuler, S. Gabriel, M. Daly, and
M.A. DePristo. 2010. The genome analysis toolkit: A MapReduce framework for analyzing next-generation DNA sequencing data. Genome Res. 20:1297-1303, doi: 10.1101/gr.107524.110.

Michelmore, R.W., I. Paran, and R.V. Kesseli. 1991. Identification of markers linked to disease-resistance genes by bulked segregant analysis: A rapid method to detect markers in specific genomic regions by using segregating populations. Proc. Natl. Acad. Sci. USA 88: 9828-9832, doi: 10.1073/pnas.88.21.9828.

Mohr, H.C. 1956. Mode of inheritance of the bushy growth characteristics in watermelon. Proc. Assoc. South Agr. Work. 53:174.

Nagai, K., K. Hirano, R.B. Angeles-Shim, and M. Ashikari. 2018. Breeding applications and molecular basis of semi-compact growth habit in rice, p. 155-176. In: T. Sasaki and H. Ashikari (eds.). Rice genomics, genetics and breeding. Springer, Singapore.

Paris, H.S., H. Nerson, and Z. Karchi. 1984. Genetics of internode length in melons. J. Hered. 75:403-406, doi: 10.1093/oxford journals.jhered.a109965.

Peng, J. 1999. Green revolution' genes encode mutant gibberellin response modulators. Nature 400:256-261, doi: 10.1038/22307.

Pitrat, M. 1991. Linkage groups in Cucumis melo L. J. Hered. 82:406-411, doi: 10.1007/BF01910546.

Robinson, R.W. and W. Mishanec. 1965. A new dwarf cucumber. Veg. Imp. Nwsl. 7:23.

Sun, T.P. 1992. Cloning the arabidopsis GA1 locus by genomic subtraction. Plant Cell 4:119-128, doi: 10.1105/tpc.4.2.119.

Teng, F., L. Zhai, R. Liu, W. Bai, L. Qiu, D. Huo, Y. Tao, Y. Zheng, and Z. Zhang. 2013. ZmGA3ox2, a candidate gene for a major QTL, qPH3.1, for plant height in maize. Plant J. 73:405-416, doi: 10.1111/ tpj. 12038 .

Thiel, T., R. Kota, I. Grosse, N. Stein, and A. Graner. 2004. SNP2CAPS: A SNP and INDEL analysis tool for CAPS marker development. Nucleic Acids Res. 32:e5, doi: 10.1093/nar/gnh006.

Wang, C., A. Qiao, X. Feng, L. Sun, P. Gao, A. Davis, S. Liu, and F. Luan. 2019. Fine mapping of lycopene content and flesh color related gene and development of molecular marker-assisted selection for flesh color in watermelon (Citrullus lanatus). Front. Plant Sci. 10:1240, doi: 10.3389/fpls.2019.01240.

Wang, K., M. Li, and H. Hakonarson. 2010. ANNOVAR: Functional annotation of genetic variants from high-throughput sequencing data. Nucleic Acids Res. 38:e164, doi: 10.1093/nar/gkq603.

Winkler, R.G. 1995. The maize dwarf3 gene encodes a cytochrome P450-mediated early step in gibberellin biosynthesis. Plant Cell 7: 1307-1317, doi: 10.1105/tpc.7.8.1307.

Xu, Y.L., L. Li, K. Wu, A.J. Peeters, D.A. Gage, and J.A. Zeevaart. 1995. The GA5 locus of Arabidopsis thaliana encodes a multifunctional gibberellin 20-oxidase: Molecular cloning and functional expression. Proc. Natl. Acad. Sci. USA 92:6640-6644, doi: 10.1073/ pnas.92.14.6640.

Yamaguchi, S. 2008. Gibberellin metabolism and its regulation. Annu. Rev. Plant Biol. 59:225-251, doi: 10.1146/annurev.arplant.59.03 2607.092804.

Yang, H., Y. Li, D. Yang, and J. Yang. 2009. A new study on shorter tendril gene of watermelon. China Cuc. Veg. 26:32-33. (In Chinese).

Zhao, S., A. Li, C. Li, H. Xia, C. Zhao, Y. Zhang, L. Hou, and X. Wang. 2017. Development and application of KASP marker for high throughput detection of AhFAD2 mutation in peanut. Electron. J. Biotechnol. 25:9-12, doi: 10.1016/j.ejbt.2016.10.010.

Zhao, T., J. Jiang, G. Liu, S. He, H. Zhang, and X. Chen. 2016. Mapping and candidate gene screening of tomato cladosporium fulvumresistant gene cf-19, based on high-throughput sequencing technology. BMC Plant Biol. 16:51-60, doi: 10.1186/s12870-016-0737-0.

Zhang, P., Y. Zhu, L. Wang, L. Chen, and S. Chen. 2015. Mining candidate genes associated with powdery mildew resistance in cucumber via super-BSA by specific length amplified fragment (slaf) sequencing. BMC Genomics 16:1058-1072, doi: 10.1186/ s12864-015-2041-z. 
Zhang, T.F., J.J. Liu, S. Liu, Z. Ding, F.S. Luan, and P. Gao. 2019. Bulked-segregant analysis identified a putative region related to short internode length in melon. HortScience 54:1293-1298, doi: 10.21273/HORTSCI14052-19.

Zheng, Y., S. Wu, Y. Bai, H. Sun, C. Jiao, S. Guo, K. Zhao, J. Blanca, Z. Zhang, S. Huang, Y. Xu, Y. Weng, M. Mazourek, U.K. Reddy, K. Ando, J.D. McCreight, A.A. Schaffer, J. Burger, Y. Tadmor, N. Katzir, X. Tang, Y. Liu, J.J. Giovannoni, K. Ling, W.P. Wechter, A. Levi, J. Garcia-Mas, R. Grumet, and Z. Fei. 2018. Cucurbit Genomics Database (CuGenDB: A central portal for comparative and functional genomics of cucurbit crops. Nucleic Acids Res. 47:1128-1136, doi: 10.1093/nar/gky944.

Zhu, H., M. Zhang, S. Sun, S. Yang, J.X. Li, H. Li, H.H. Yang, K.G. Zhang, J.B. Hu, D.M. Liu, and L.M. Yang. 2019. A single nucleotide deletion in an $\mathrm{ABC}$ transporter gene leads to a dwarf phenotype in watermelon. Front. Plant Sci. 10:1399, doi: 10.3389/fpls.2019.01399.

Zhang, W., L.Q. Zeng, Y.Y. Li, M. Gui, R. Chen, X.Q. Huang, and Z.Q. Cheng. 2006. Isolation of total RNA from the different tissue of Gerbera jamesonii Bolu. Xi Nan Nong Ye Xue Bao 19:705-708, doi: 10.3321/j.issn:0890-5487.2006.03.009. (In Chinese). 
Supplemental Table 1. Developed kompetitive allele-specific PCR (KASP) markers used for primary mapping and fine mapping of Cldw gene, respectively.

KASP marker used for primary mapping

\begin{tabular}{|c|c|c|}
\hline \# & KASP marker & Primer sequence \\
\hline \multirow[t]{3}{*}{1} & K1198013-AlleleX & TAATTGTAACTTTTAGCCTTTCAGTCCT \\
\hline & K1198013-AlleleY & TAATTGTAACTTTTAGCCTTTCAGTCCA \\
\hline & K1198013-Common & TAATGCATACTCATAACTTTGTTTACCTAAATTGA \\
\hline \multirow[t]{3}{*}{2} & K1421609-AlleleX & GAAGGTGACCAAGTTCATGCTCCTCTCTCTCССССТСССТT \\
\hline & K1421609-AlleleY & GAAGGTCGGAGTCAACGGATTCTCTCTCTCССССТСССТC \\
\hline & K1421609-Common & ACACCAATTGAAATCAAACTTAAAACTTAATTCAA \\
\hline \multirow[t]{3}{*}{3} & K1692577-AlleleX & GAAGGTGACCAAGTTCATGCTGGTGTACTAAGTACAGCACAAAGGAT \\
\hline & K1692577-AlleleY & GAAGGTCGGAGTCAACGGATTGTGTACTAAGTACAGCACAAAGGAG \\
\hline & K1692577-Common & AAAGTAAAGAGTATGATTTGGAACCTGGATT \\
\hline \multirow[t]{3}{*}{4} & K1906994-AlleleX & GAAGGTGACCAAGTTCATGCTTTACACTTTGAGTACATATCAATAAGAGAATTT \\
\hline & K1906994-AlleleY & GAAGGTCGGAGTCAACGGATTACACTTTGAGTACATATCAATAAGAGAATTC \\
\hline & K1906994-Common & ACTGGCCTCATATCACATGTCATAGTAT \\
\hline \multirow[t]{3}{*}{5} & K2418327-AlleleX & GAAGGTGACCAAGTTCATGCTCAGTACCATTGAATATAAATGTTTCTTTAAGC \\
\hline & K2418327-AlleleY & GAAGGTCGGAGTCAACGGATTTCAGTACCATTGAATATAAATGTTTCTTTAAGA \\
\hline & K2418327-Common & TGATAGGTTTTCGGTTGAAATTAGTTTATCAATT \\
\hline \multirow[t]{3}{*}{6} & K2739100-AlleleX & GAAGGTGACCAAGTTCATGCTTCTTTAGGGTCCTACAAATTACCAAAC \\
\hline & K2739100-AlleleY & GAAGGTCGGAGTCAACGGATTTCTTTAGGGTCCTACAAATTACCAAAG \\
\hline & K2739100-Common & AAGGGGTGGGTTCTGAATGGTAAAATTT \\
\hline \multirow[t]{3}{*}{7} & K2836103-AlleleX & CCTGGTGATAGACTCCTAACATTG \\
\hline & K2836103-AlleleY & CCCTGGTGATAGACTCCTAACATTA \\
\hline & K2836103-Common & TTTTAGGGAAAATAATTAAGTGCACAACACCA \\
\hline \multirow[t]{3}{*}{8} & K3014648-AlleleX & GAAGGTGACCAAGTTCATGCTGTTGAGTTACTTAACACTAATTTATGAGACA \\
\hline & K3014648-AlleleY & GAAGGTCGGAGTCAACGGATTTTGAGTTACTTAACACTAATTTATGAGACC \\
\hline & K3014648-Common & CACTTTAACTTTTGAAATAACACGAGGTGTTTA \\
\hline \multirow[t]{3}{*}{9} & K3319944-AlleleX & GAAGGTGACCAAGTTCATGCTATTTTGTGTCTCTCCCTCACTCTGA \\
\hline & K3319944-AlleleY & GAAGGTCGGAGTCAACGGATTATTTTGTGTCTCTCCCTCACTCTGT \\
\hline & K3319944-Common & CCAAAATGAATTCTCCAAAAATGACAGCTATATT \\
\hline \multirow[t]{3}{*}{10} & K3673471-AlleleX & GAAGGTGACCAAGTTCATGCTATTAATCTCTCTTTTTGACACCATTGACTA \\
\hline & K3673471-AlleleY & GAAGGTCGGAGTCAACGGATTTAATCTCTCTTTTTGACACCATTGACTG \\
\hline & K3673471-Common & TCGTCATCCGTTGGAATGTTAATCTCTT \\
\hline \multirow[t]{3}{*}{11} & K1692577-AlleleX & GAAGGTGACCAAGTTCATGCTGGTGTACTAAGTACAGCACAAAGGAT \\
\hline & K1692577-AlleleY & GAAGGTCGGAGTCAACGGATTGTGTACTAAGTACAGCACAAAGGAG \\
\hline & K1692577-Common & AAAGTAAAGAGTATGATTTGGAACCTGGATT \\
\hline \multirow[t]{3}{*}{12} & K1764972-AlleleX & GAAATTGACATGACTTTTCTACCTTGAAA \\
\hline & K1764972-AlleleY & AAATTGACATGACTTTTCTACCTTGAAG \\
\hline & K1764972-Common & CATAAACCTTCAAGATTTGTGTCCGTTAAC \\
\hline \multirow[t]{3}{*}{13} & K1822206-AlleleX & GAAGGTGACCAAGTTCATGCTTTAATTTGCTACCTCTCCAATAACTAAATAAAT \\
\hline & K1822206-AlleleY & GAAGGTCGGAGTCAACGGATTAATTTGCTACCTCTCCAATAACTAAATAAAC \\
\hline & K1822206-Common & TTTATTTTGATTTTTTGCAAGTGGCCCATATATA \\
\hline \multirow[t]{3}{*}{14} & K1865395-AlleleX & GAAGGTGACCAAGTTCATGCTGGTGCCTACAAGACCTTATTCTG \\
\hline & K1865395-AlleleY & GAAGGTCGGAGTCAACGGATTGGTGCCTACAAGACCTTATTCTC \\
\hline & K1865395-Common & GGATTGGCACAGGCATGGAGCAA \\
\hline \multirow[t]{3}{*}{15} & K1906994-AlleleX & GAAGGTGACCAAGTTCATGCTTTACACTTTGAGTACATATCAATAAGAGAATTT \\
\hline & K1906994-AlleleY & GAAGGTCGGAGTCAACGGATTACACTTTGAGTACATATCAATAAGAGAATTC \\
\hline & K1906994-Common & ACTGGCCTCATATCACATGTCATAGTAT \\
\hline \multirow[t]{3}{*}{16} & KCla015408-AlleleX & GAAGGTGACCAAGTTCATGCTAACTTTTTAACGACACACTCTCATCTC \\
\hline & KCla015408-AlleleY & GAAGGTCGGAGTCAACGGATTGAACTTTTTAACGACACACTCTCATCTA \\
\hline & KCla015408-Common & CTAACAGTCCAGTGAGAGGAGTGTA \\
\hline
\end{tabular}


Supplemental Table 2. Bulked segregant analysis (BSA) based delta $(\Delta)$ single nucleotide polymorphism (SNP)_index (1.2-3.6 Mb) of chromosome 9; Standard type bulk is abbreviated by S-bulk and Dwarf type bulk is abbreviated by D-bulk.

\begin{tabular}{|c|c|c|c|c|}
\hline Chr. & Physical location & SNP index, S-bulk (\%) & SNP index, D-bulk (\%) & $\Delta \mathrm{SNP}$ index \\
\hline 9 & 1200466 & 0 & 100 & 1 \\
\hline 9 & 1200515 & 0 & 100 & 1 \\
\hline 9 & 1202354 & 0 & 100 & 1 \\
\hline 9 & 1202588 & 0 & 100 & 1 \\
\hline 9 & 1203077 & 0 & 100 & 1 \\
\hline 9 & 1207298 & 0 & 100 & 1 \\
\hline 9 & 1210019 & 3.57 & 100 & 0.9643 \\
\hline 9 & 1210670 & 0 & 100 & 1 \\
\hline 9 & 1211013 & 0 & 100 & 1 \\
\hline 9 & 1213344 & 0 & 100 & 1 \\
\hline 9 & 1213345 & 0 & 100 & 1 \\
\hline 9 & 1214268 & 0 & 100 & 1 \\
\hline 9 & 1216316 & 0 & 100 & 1 \\
\hline 9 & 1216877 & 3.7 & 100 & 0.963 \\
\hline 9 & 1216893 & 3.85 & 100 & 0.9615 \\
\hline 9 & 1217981 & 0 & 100 & 1 \\
\hline 9 & 1218113 & 2.78 & 100 & 0.9722 \\
\hline 9 & 1221027 & 0 & 100 & 1 \\
\hline 9 & 1221165 & 4.55 & 100 & 0.9545 \\
\hline 9 & 1222501 & 0 & 100 & 1 \\
\hline 9 & 1223078 & 0 & 100 & 1 \\
\hline 9 & 1227589 & 0 & 100 & 1 \\
\hline 9 & 1229062 & 0 & 100 & 1 \\
\hline 9 & 1229805 & 0 & 100 & 1 \\
\hline 9 & 1233475 & 0 & 20 & 0.2 \\
\hline 9 & 1233499 & 0 & 20 & 0.2 \\
\hline 9 & 1233508 & 0 & 20 & 0.2 \\
\hline 9 & 1233516 & 0 & 20 & 0.2 \\
\hline 9 & 1233529 & 0 & 20 & 0.2 \\
\hline 9 & 1234393 & 0 & 20 & 0.2 \\
\hline 9 & 1234404 & 0 & 20 & 0.2 \\
\hline 9 & 1234405 & 0 & 20 & 0.2 \\
\hline 9 & 1234427 & 0 & 20 & 0.2 \\
\hline 9 & 1234432 & 0 & 20 & 0.2 \\
\hline 9 & 1234437 & 0 & 20 & 0.2 \\
\hline 9 & 1234465 & 0 & 20 & 0.2 \\
\hline 9 & 1237087 & 0 & 100 & 1 \\
\hline 9 & 1237100 & 0 & 100 & 1 \\
\hline 9 & 1237132 & 0 & 100 & 1 \\
\hline 9 & 1238498 & 0 & 100 & 1 \\
\hline 9 & 1238648 & 0 & 100 & 1 \\
\hline 9 & 1241236 & 0 & 100 & 1 \\
\hline 9 & 1242093 & 0 & 100 & 1 \\
\hline 9 & 1242617 & 0 & 100 & 1 \\
\hline 9 & 1245103 & 0 & 100 & 1 \\
\hline 9 & 1249217 & 0 & 100 & 1 \\
\hline 9 & 1249584 & 0 & 100 & 1 \\
\hline 9 & 1250335 & 0 & 100 & 1 \\
\hline 9 & 1250400 & 0 & 100 & 1 \\
\hline 9 & 1251381 & 0 & 100 & 1 \\
\hline 9 & 1251427 & 0 & 22.22 & 0.2222 \\
\hline 9 & 1251447 & 0 & 22.22 & 0.2222 \\
\hline 9 & 1255961 & 0 & 28.57 & 0.2857 \\
\hline 9 & 1259898 & 0 & 100 & 1 \\
\hline 9 & 1263117 & 0 & 100 & 1 \\
\hline 9 & 1263159 & 0 & 100 & 1 \\
\hline
\end{tabular}

(Continued on next page) 
(Continued) Supplemental Table 2. Bulked segregant analysis (BSA) based delta $(\Delta)$ single nucleotide polymorphism (SNP)_index (1.2-3.6 Mb) of chromosome 9; Standard type bulk is abbreviated by S-bulk and Dwarf type bulk is abbreviated by D-bulk.

\begin{tabular}{|c|c|c|c|c|}
\hline Chr. & Physical location & SNP index, S-bulk (\%) & SNP index, D-bulk (\%) & $\Delta \mathrm{SNP}$ index \\
\hline 9 & 1263359 & 2.78 & 100 & 0.9722 \\
\hline 9 & 1264606 & 0 & 100 & 1 \\
\hline 9 & 1264959 & 0 & 100 & 1 \\
\hline 9 & 1267598 & 0 & 100 & 1 \\
\hline 9 & 1267651 & 0 & 100 & 1 \\
\hline 9 & 1268909 & 0 & 45.45 & 0.4545 \\
\hline 9 & 1268924 & 0 & 41.67 & 0.4167 \\
\hline 9 & 1269000 & 0 & 42.86 & 0.4286 \\
\hline 9 & 1269152 & 32.26 & 52.63 & 0.2037 \\
\hline 9 & 1269187 & 21.74 & 33.33 & 0.1159 \\
\hline 9 & 1269269 & 2.78 & 26.67 & 0.2389 \\
\hline 9 & 1269271 & 0 & 30.77 & 0.3077 \\
\hline 9 & 1269519 & 38.71 & 0 & -0.3871 \\
\hline 9 & 1270085 & 15.38 & 47.06 & 0.3168 \\
\hline 9 & 1270088 & 13.89 & 47.06 & 0.3317 \\
\hline 9 & 1270112 & 16.13 & 44.44 & 0.2831 \\
\hline 9 & 1270118 & 15.62 & 50 & 0.3438 \\
\hline 9 & 1270153 & 7.41 & 42.86 & 0.3545 \\
\hline 9 & 1270345 & 33.33 & 52.94 & 0.1961 \\
\hline 9 & 1270440 & 28.57 & 37.5 & 0.0893 \\
\hline 9 & 1270461 & 20.69 & 33.33 & 0.1264 \\
\hline 9 & 1270523 & 25 & 100 & 0.75 \\
\hline 9 & 1270675 & 7.32 & 100 & 0.9268 \\
\hline 9 & 1271905 & 0 & 100 & 1 \\
\hline 9 & 1272411 & 4.55 & 100 & 0.9545 \\
\hline 9 & 1273941 & 0 & 100 & 1 \\
\hline 9 & 1278061 & 0 & 20 & 0.2 \\
\hline 9 & 1279397 & 0 & 100 & 1 \\
\hline 9 & 1279725 & 0 & 100 & 1 \\
\hline 9 & 1280289 & 0 & 100 & 1 \\
\hline 9 & 1282818 & 0 & 100 & 1 \\
\hline 9 & 1283286 & 0 & 100 & 1 \\
\hline 9 & 1283910 & 0 & 100 & 1 \\
\hline 9 & 1285656 & 0 & 22.22 & 0.2222 \\
\hline 9 & 1287347 & 2.94 & 85.71 & 0.8277 \\
\hline 9 & 1295253 & 0 & 100 & 1 \\
\hline 9 & 1299889 & 0 & 25 & 0.25 \\
\hline 9 & 1299892 & 0 & 25 & 0.25 \\
\hline 9 & 1299894 & 0 & 25 & 0.25 \\
\hline 9 & 1310963 & 0 & 100 & 1 \\
\hline 9 & 1315962 & 0 & 100 & 1 \\
\hline 9 & 1315974 & 0 & 100 & 1 \\
\hline 9 & 1318316 & 0 & 20 & 0.2 \\
\hline 9 & 1318334 & 0 & 20 & 0.2 \\
\hline 9 & 1322711 & 0 & 100 & 1 \\
\hline 9 & 1329270 & 0 & 100 & 1 \\
\hline 9 & 1329276 & 0 & 100 & 1 \\
\hline 9 & 1329339 & 0 & 100 & 1 \\
\hline 9 & 1329607 & 0 & 100 & 1 \\
\hline 9 & 1332229 & 0 & 100 & 1 \\
\hline 9 & 1341857 & 0 & 100 & 1 \\
\hline 9 & 1347465 & 0 & 22.22 & 0.2222 \\
\hline 9 & 1347486 & 0 & 20 & 0.2 \\
\hline 9 & 1347490 & 0 & 20 & 0.2 \\
\hline 9 & 1347500 & 0 & 22.22 & 0.2222 \\
\hline 9 & 1379597 & 0 & 20 & 0.2 \\
\hline
\end{tabular}


(Continued) Supplemental Table 2. Bulked segregant analysis (BSA) based delta $(\Delta)$ single nucleotide polymorphism (SNP)_index (1.2-3.6 Mb) of chromosome 9; Standard type bulk is abbreviated by S-bulk and Dwarf type bulk is abbreviated by D-bulk.

\begin{tabular}{|c|c|c|c|c|}
\hline Chr. & Physical location & SNP index, S-bulk (\%) & SNP index, D-bulk (\%) & $\Delta \mathrm{SNP}$ index \\
\hline 9 & 1396859 & 0 & 100 & 1 \\
\hline 9 & 1403535 & 0 & 100 & 1 \\
\hline 9 & 1418686 & 0 & 100 & 1 \\
\hline 9 & 1421609 & 0 & 100 & 1 \\
\hline 9 & 1425301 & 0 & 100 & 1 \\
\hline 9 & 1427156 & 10 & 100 & 0.9 \\
\hline 9 & 1432267 & 0 & 100 & 1 \\
\hline 9 & 1432934 & 0 & 100 & 1 \\
\hline 9 & 1433855 & 0 & 100 & 1 \\
\hline 9 & 1440287 & 0 & 22.22 & 0.2222 \\
\hline 9 & 1440334 & 0 & 100 & 1 \\
\hline 9 & 1455072 & 0 & 100 & 1 \\
\hline 9 & 1460000 & 0 & 100 & 1 \\
\hline 9 & 1460545 & 0 & 100 & 1 \\
\hline 9 & 1462240 & 0 & 100 & 1 \\
\hline 9 & 1462486 & 0 & 100 & 1 \\
\hline 9 & 1462487 & 0 & 100 & 1 \\
\hline 9 & 1465289 & 0 & 20 & 0.2 \\
\hline 9 & 1465307 & 0 & 22.22 & 0.2222 \\
\hline 9 & 1465315 & 0 & 20 & 0.2 \\
\hline 9 & 1466958 & 0 & 100 & 1 \\
\hline 9 & 1468030 & 3.85 & 100 & 0.9615 \\
\hline 9 & 1469202 & 0 & 100 & 1 \\
\hline 9 & 1469260 & 0 & 100 & 1 \\
\hline 9 & 1469437 & 0 & 100 & 1 \\
\hline 9 & 1470078 & 0 & 100 & 1 \\
\hline 9 & 1470174 & 0 & 100 & 1 \\
\hline 9 & 1470178 & 0 & 100 & 1 \\
\hline 9 & 1473939 & 0 & 22.22 & 0.2222 \\
\hline 9 & 1473993 & 0 & 22.22 & 0.2222 \\
\hline 9 & 1473996 & 0 & 22.22 & 0.2222 \\
\hline 9 & 1476702 & 0 & 100 & 1 \\
\hline 9 & 1476917 & 0 & 100 & 1 \\
\hline 9 & 1477675 & 0 & 100 & 1 \\
\hline 9 & 1480622 & 4.35 & 100 & 0.9565 \\
\hline 9 & 1481379 & 0 & 100 & 1 \\
\hline 9 & 1481478 & 0 & 100 & 1 \\
\hline 9 & 1482832 & 0 & 100 & 1 \\
\hline 9 & 1490955 & 0 & 100 & 1 \\
\hline 9 & 1493286 & 0 & 88.89 & 0.8889 \\
\hline 9 & 1500483 & 0 & 25 & 0.25 \\
\hline 9 & 1500531 & 0 & 25 & 0.25 \\
\hline 9 & 1502477 & 0 & 25 & 0.25 \\
\hline 9 & 1502485 & 0 & 22.22 & 0.2222 \\
\hline 9 & 1502487 & 0 & 22.22 & 0.2222 \\
\hline 9 & 1502493 & 0 & 20 & 0.2 \\
\hline 9 & 1502759 & 0 & 100 & 1 \\
\hline 9 & 1503870 & 0 & 100 & 1 \\
\hline 9 & 1519190 & 66.67 & 0 & -0.6667 \\
\hline 9 & 1540966 & 0 & 100 & 1 \\
\hline 9 & 1540992 & 0 & 100 & 1 \\
\hline 9 & 1547048 & 0 & 100 & 1 \\
\hline 9 & 1550425 & 3.33 & 100 & 0.9667 \\
\hline 9 & 1550689 & 0 & 100 & 1 \\
\hline 9 & 1551121 & 0 & 100 & 1 \\
\hline 9 & 1580462 & 0 & 22.22 & 0.2222 \\
\hline
\end{tabular}


(Continued) Supplemental Table 2. Bulked segregant analysis (BSA) based delta $(\Delta)$ single nucleotide polymorphism (SNP)_index (1.2-3.6 Mb) of chromosome 9; Standard type bulk is abbreviated by S-bulk and Dwarf type bulk is abbreviated by D-bulk.

\begin{tabular}{|c|c|c|c|c|}
\hline Chr. & Physical location & SNP index, S-bulk (\%) & SNP index, D-bulk (\%) & $\Delta \mathrm{SNP}$ index \\
\hline 9 & 1580486 & 0 & 25 & 0.25 \\
\hline 9 & 1580495 & 0 & 22.22 & 0.2222 \\
\hline 9 & 1580503 & 0 & 22.22 & 0.2222 \\
\hline 9 & 1580504 & 0 & 20 & 0.2 \\
\hline 9 & 1580515 & 0 & 20 & 0.2 \\
\hline 9 & 1594752 & 0 & 22.22 & 0.2222 \\
\hline 9 & 1605613 & 0 & 25 & 0.25 \\
\hline 9 & 1605620 & 0 & 25 & 0.25 \\
\hline 9 & 1605628 & 0 & 25 & 0.25 \\
\hline 9 & 1605654 & 0 & 25 & 0.25 \\
\hline 9 & 1605661 & 0 & 25 & 0.25 \\
\hline 9 & 1605669 & 0 & 33.33 & 0.3333 \\
\hline 9 & 1616570 & 0 & 100 & 1 \\
\hline 9 & 1633355 & 0 & 100 & 1 \\
\hline 9 & 1633399 & 0 & 100 & 1 \\
\hline 9 & 1634492 & 0 & 50 & 0.5 \\
\hline 9 & 1634493 & 7.14 & 75 & 0.6786 \\
\hline 9 & 1637805 & 0 & 100 & 1 \\
\hline 9 & 1638646 & 0 & 22.22 & 0.2222 \\
\hline 9 & 1639214 & 0 & 37.5 & 0.375 \\
\hline 9 & 1639231 & 0 & 100 & 1 \\
\hline 9 & 1640886 & 0 & 100 & 1 \\
\hline 9 & 1642416 & 0 & 20 & 0.2 \\
\hline 9 & 1643601 & 0 & 100 & 1 \\
\hline 9 & 1643707 & 0 & 100 & 1 \\
\hline 9 & 1646455 & 0 & 100 & 1 \\
\hline 9 & 1646687 & 5.88 & 100 & 0.9412 \\
\hline 9 & 1647005 & 0 & 100 & 1 \\
\hline 9 & 1648226 & 0 & 100 & 1 \\
\hline 9 & 1649594 & 0 & 100 & 1 \\
\hline 9 & 1650368 & 0 & 100 & 1 \\
\hline 9 & 1650393 & 0 & 100 & 1 \\
\hline 9 & 1650833 & 5.56 & 100 & 0.9444 \\
\hline 9 & 1651840 & 3.45 & 100 & 0.9655 \\
\hline 9 & 1652670 & 0 & 100 & 1 \\
\hline 9 & 1653586 & 0 & 92.31 & 0.9231 \\
\hline 9 & 1653721 & 0 & 28.57 & 0.2857 \\
\hline 9 & 1654080 & 0 & 100 & 1 \\
\hline 9 & 1654242 & 0 & 100 & 1 \\
\hline 9 & 1659543 & 0 & 100 & 1 \\
\hline 9 & 1659792 & 0 & 100 & 1 \\
\hline 9 & 1662802 & 0 & 100 & 1 \\
\hline 9 & 1665251 & 0 & 100 & 1 \\
\hline 9 & 1668099 & 0 & 100 & 1 \\
\hline 9 & 1669258 & 0 & 100 & 1 \\
\hline 9 & 1669347 & 0 & 90 & 0.9 \\
\hline 9 & 1669367 & 0 & 88.89 & 0.8889 \\
\hline 9 & 1674704 & 0 & 100 & 1 \\
\hline 9 & 1674731 & 4.17 & 100 & 0.9583 \\
\hline 9 & 1676018 & 0 & 100 & 1 \\
\hline 9 & 1692577 & 2.94 & 100 & 0.9706 \\
\hline 9 & 1700132 & 0 & 20 & 0.2 \\
\hline 9 & 1700133 & 0 & 25 & 0.25 \\
\hline 9 & 1700138 & 0 & 22.22 & 0.2222 \\
\hline 9 & 1700151 & 0 & 20 & 0.2 \\
\hline 9 & 1700156 & 0 & 20 & 0.2 \\
\hline
\end{tabular}


(Continued) Supplemental Table 2. Bulked segregant analysis (BSA) based delta $(\Delta)$ single nucleotide polymorphism (SNP)_index (1.2-3.6 Mb) of chromosome 9; Standard type bulk is abbreviated by S-bulk and Dwarf type bulk is abbreviated by D-bulk.

\begin{tabular}{|c|c|c|c|c|}
\hline Chr. & Physical location & SNP index, S-bulk (\%) & SNP index, D-bulk (\%) & $\Delta \mathrm{SNP}$ index \\
\hline 9 & 1735168 & 66.67 & 40 & -0.2667 \\
\hline 9 & 1738954 & 0 & 22.22 & 0.2222 \\
\hline 9 & 1739284 & 0 & 20 & 0.2 \\
\hline 9 & 1763379 & 0 & 100 & 1 \\
\hline 9 & 1764972 & 0 & 100 & 1 \\
\hline 9 & 1765622 & 0 & 100 & 1 \\
\hline 9 & 1776634 & 2.7 & 100 & 0.973 \\
\hline 9 & 1776848 & 0 & 100 & 1 \\
\hline 9 & 1777113 & 0 & 100 & 1 \\
\hline 9 & 1778400 & 0 & 100 & 1 \\
\hline 9 & 1778537 & 0 & 100 & 1 \\
\hline 9 & 1778548 & 0 & 100 & 1 \\
\hline 9 & 1778804 & 0 & 90 & 0.9 \\
\hline 9 & 1778816 & 0 & 20 & 0.2 \\
\hline 9 & 1779210 & 0 & 100 & 1 \\
\hline 9 & 1779319 & 0 & 100 & 1 \\
\hline 9 & 1779836 & 0 & 100 & 1 \\
\hline 9 & 1787778 & 0 & 100 & 1 \\
\hline 9 & 1788598 & 0 & 100 & 1 \\
\hline 9 & 1789514 & 0 & 100 & 1 \\
\hline 9 & 1789570 & 0 & 26.67 & 0.2667 \\
\hline 9 & 1789598 & 0 & 30.77 & 0.3077 \\
\hline 9 & 1789604 & 0 & 69.23 & 0.6923 \\
\hline 9 & 1789626 & 0 & 40 & 0.4 \\
\hline 9 & 1790066 & 0 & 100 & 1 \\
\hline 9 & 1790090 & 0 & 100 & 1 \\
\hline 9 & 1791709 & 0 & 100 & 1 \\
\hline 9 & 1793357 & 0 & 20 & 0.2 \\
\hline 9 & 1794386 & 0 & 20 & 0.2 \\
\hline 9 & 1794641 & 0 & 100 & 1 \\
\hline 9 & 1798900 & 0 & 100 & 1 \\
\hline 9 & 1801487 & 0 & 100 & 1 \\
\hline 9 & 1803967 & 0 & 100 & 1 \\
\hline 9 & 1804244 & 0 & 100 & 1 \\
\hline 9 & 1805434 & 0 & 100 & 1 \\
\hline 9 & 1810044 & 0 & 100 & 1 \\
\hline 9 & 1812630 & 50 & 37.5 & -0.125 \\
\hline 9 & 1812634 & 0 & 25 & 0.25 \\
\hline 9 & 1816187 & 0 & 100 & 1 \\
\hline 9 & 1816207 & 0 & 100 & 1 \\
\hline 9 & 1816368 & 0 & 100 & 1 \\
\hline 9 & 1821818 & 0 & 100 & 1 \\
\hline 9 & 1822112 & 0 & 100 & 1 \\
\hline 9 & 1822206 & 0 & 100 & 1 \\
\hline 9 & 1857557 & 0 & 28.57 & 0.2857 \\
\hline 9 & 1857578 & 0 & 25 & 0.25 \\
\hline 9 & 1857581 & 0 & 25 & 0.25 \\
\hline 9 & 1865395 & 0 & 100 & 1 \\
\hline 9 & 1870253 & 0 & 100 & 1 \\
\hline 9 & 1871033 & 0 & 100 & 1 \\
\hline 9 & 1872994 & 0 & 100 & 1 \\
\hline 9 & 1873184 & 5.56 & 75 & 0.6944 \\
\hline 9 & 1887997 & 0 & 100 & 1 \\
\hline 9 & 1889879 & 0 & 20 & 0.2 \\
\hline 9 & 1897481 & 0 & 100 & 1 \\
\hline 9 & 1899283 & 0 & 100 & 1 \\
\hline
\end{tabular}


(Continued) Supplemental Table 2. Bulked segregant analysis (BSA) based delta $(\Delta)$ single nucleotide polymorphism (SNP)_index (1.2-3.6 Mb) of chromosome 9; Standard type bulk is abbreviated by S-bulk and Dwarf type bulk is abbreviated by D-bulk.

\begin{tabular}{|c|c|c|c|c|}
\hline Chr. & Physical location & SNP index, S-bulk (\%) & SNP index, D-bulk (\%) & $\Delta \mathrm{SNP}$ index \\
\hline 9 & 1899737 & 0 & 100 & 1 \\
\hline 9 & 1899773 & 0 & 100 & 1 \\
\hline 9 & 1900347 & 0 & 100 & 1 \\
\hline 9 & 1902861 & 0 & 22.22 & 0.2222 \\
\hline 9 & 1902894 & 0 & 25 & 0.25 \\
\hline 9 & 1902899 & 0 & 28.57 & 0.2857 \\
\hline 9 & 1903507 & 0 & 100 & 1 \\
\hline 9 & 1906231 & 0 & 100 & 1 \\
\hline 9 & 1906579 & 0 & 28.57 & 0.2857 \\
\hline 9 & 1906592 & 0 & 25 & 0.25 \\
\hline 9 & 1906994 & 0 & 100 & 1 \\
\hline 9 & 1907164 & 0 & 20 & 0.2 \\
\hline 9 & 1907366 & 0 & 100 & 1 \\
\hline 9 & 1907846 & 0 & 20 & 0.2 \\
\hline 9 & 1913510 & 0 & 100 & 1 \\
\hline 9 & 1913960 & 0 & 100 & 1 \\
\hline 9 & 1914093 & 4.35 & 100 & 0.9565 \\
\hline 9 & 1934427 & 0 & 28.57 & 0.2857 \\
\hline 9 & 1936940 & 0 & 20 & 0.2 \\
\hline 9 & 1972173 & 0 & 20 & 0.2 \\
\hline 9 & 1976411 & 0 & 20 & 0.2 \\
\hline 9 & 1984231 & 0 & 23.08 & 0.2308 \\
\hline 9 & 1984235 & 0 & 21.43 & 0.2143 \\
\hline 9 & 1984267 & 0 & 33.33 & 0.3333 \\
\hline 9 & 1984279 & 0 & 37.5 & 0.375 \\
\hline 9 & 1991587 & 0 & 22.22 & 0.2222 \\
\hline 9 & 1991591 & 0 & 20 & 0.2 \\
\hline 9 & 1991599 & 0 & 20 & 0.2 \\
\hline 9 & 1991652 & 0 & 22.22 & 0.2222 \\
\hline 9 & 1991697 & 0 & 25 & 0.25 \\
\hline 9 & 1991701 & 0 & 25 & 0.25 \\
\hline 9 & 1991703 & 0 & 25 & 0.25 \\
\hline 9 & 1994055 & 63.04 & 70 & 0.0696 \\
\hline 9 & 1994226 & 28.89 & 41.18 & 0.1229 \\
\hline 9 & 1994230 & 28.26 & 36.84 & 0.0858 \\
\hline 9 & 1994470 & 52.78 & 66.67 & 0.1389 \\
\hline 9 & 1999557 & 0 & 22.22 & 0.2222 \\
\hline 9 & 2023135 & 0 & 22.22 & 0.2222 \\
\hline 9 & 2023174 & 0 & 20 & 0.2 \\
\hline 9 & 2023191 & 0 & 20 & 0.2 \\
\hline 9 & 2023214 & 0 & 25 & 0.25 \\
\hline 9 & 2044420 & 0 & 22.22 & 0.2222 \\
\hline 9 & 2044527 & 0 & 20 & 0.2 \\
\hline 9 & 2062274 & 0 & 22.22 & 0.2222 \\
\hline 9 & 2075664 & 0 & 25 & 0.25 \\
\hline 9 & 2080943 & 0 & 21.43 & 0.2143 \\
\hline 9 & 2081000 & 0 & 25 & 0.25 \\
\hline 9 & 2081114 & 0 & 25 & 0.25 \\
\hline 9 & 2081656 & 0 & 20 & 0.2 \\
\hline 9 & 2102099 & 0 & 22.22 & 0.2222 \\
\hline 9 & 2109019 & 0 & 28.57 & 0.2857 \\
\hline 9 & 2141991 & 0 & 22.22 & 0.2222 \\
\hline 9 & 2188105 & 0 & 22.22 & 0.2222 \\
\hline 9 & 2194903 & 0 & 25 & 0.25 \\
\hline 9 & 2195632 & 0 & 22.22 & 0.2222 \\
\hline 9 & 2210030 & 0 & 25 & 0.25 \\
\hline
\end{tabular}


(Continued) Supplemental Table 2. Bulked segregant analysis (BSA) based delta $(\Delta)$ single nucleotide polymorphism (SNP)_index (1.2-3.6 Mb) of chromosome 9; Standard type bulk is abbreviated by S-bulk and Dwarf type bulk is abbreviated by D-bulk.

\begin{tabular}{|c|c|c|c|c|}
\hline Chr. & Physical location & SNP index, S-bulk (\%) & SNP index, D-bulk (\%) & $\Delta \mathrm{SNP}$ index \\
\hline 9 & 2210057 & 0 & 22.22 & 0.2222 \\
\hline 9 & 2210060 & 0 & 25 & 0.25 \\
\hline 9 & 2210072 & 0 & 28.57 & 0.2857 \\
\hline 9 & 2217055 & 0 & 22.22 & 0.2222 \\
\hline 9 & 2217059 & 0 & 22.22 & 0.2222 \\
\hline 9 & 2222104 & 0 & 25 & 0.25 \\
\hline 9 & 2237349 & 0 & 42.86 & 0.4286 \\
\hline 9 & 2237359 & 3.23 & 37.5 & 0.3427 \\
\hline 9 & 2261769 & 0 & 22.22 & 0.2222 \\
\hline 9 & 2262360 & 0 & 22.22 & 0.2222 \\
\hline 9 & 2267479 & 0 & 22.22 & 0.2222 \\
\hline 9 & 2271001 & 100 & 88.89 & -0.1111 \\
\hline 9 & 2271002 & 100 & 90 & -0.1 \\
\hline 9 & 2271003 & 100 & 90 & -0.1 \\
\hline 9 & 2271107 & 100 & 92.86 & -0.0714 \\
\hline 9 & 2282120 & 0 & 88.89 & 0.8889 \\
\hline 9 & 2282144 & 0 & 92.31 & 0.9231 \\
\hline 9 & 2282145 & 0 & 92.31 & 0.9231 \\
\hline 9 & 2282293 & 0 & 90.7 & 0.907 \\
\hline 9 & 2282298 & 0 & 100 & 1 \\
\hline 9 & 2282330 & 0 & 94.59 & 0.9459 \\
\hline 9 & 2282338 & 0 & 94.12 & 0.9412 \\
\hline 9 & 2282347 & 0 & 92 & 0.92 \\
\hline 9 & 2282348 & 0 & 92 & 0.92 \\
\hline 9 & 2282360 & 0 & 90.48 & 0.9048 \\
\hline 9 & 2298241 & 0 & 20 & 0.2 \\
\hline 9 & 2319055 & 0 & 21.43 & 0.2143 \\
\hline 9 & 2319066 & 0 & 23.08 & 0.2308 \\
\hline 9 & 2319067 & 0 & 23.08 & 0.2308 \\
\hline 9 & 2319068 & 0 & 23.08 & 0.2308 \\
\hline 9 & 2319094 & 0 & 30.77 & 0.3077 \\
\hline 9 & 2319101 & 0 & 30 & 0.3 \\
\hline 9 & 2319107 & 0 & 37.5 & 0.375 \\
\hline 9 & 2319108 & 0 & 30 & 0.3 \\
\hline 9 & 2319153 & 0 & 23.08 & 0.2308 \\
\hline 9 & 2319158 & 0 & 23.08 & 0.2308 \\
\hline 9 & 2319160 & 0 & 27.27 & 0.2727 \\
\hline 9 & 2319176 & 0 & 20 & 0.2 \\
\hline 9 & 2319182 & 0 & 20 & 0.2 \\
\hline 9 & 2319186 & 0 & 20 & 0.2 \\
\hline 9 & 2330895 & 0 & 20 & 0.2 \\
\hline 9 & 2330913 & 0 & 22.22 & 0.2222 \\
\hline 9 & 2330934 & 0 & 22.22 & 0.2222 \\
\hline 9 & 2352557 & 0 & 25 & 0.25 \\
\hline 9 & 2352578 & 0 & 22.22 & 0.2222 \\
\hline 9 & 2352583 & 0 & 22.22 & 0.2222 \\
\hline 9 & 2367894 & 0 & 90 & 0.9 \\
\hline 9 & 2371243 & 100 & 0 & -1 \\
\hline 9 & 2372111 & 100 & 0 & -1 \\
\hline 9 & 2375950 & 0 & 100 & 1 \\
\hline 9 & 2380093 & 0 & 33.33 & 0.3333 \\
\hline 9 & 2381685 & 91.67 & 100 & 0.0833 \\
\hline 9 & 2399954 & 0 & 100 & 1 \\
\hline 9 & 2400494 & 0 & 100 & 1 \\
\hline 9 & 2401274 & 0 & 100 & 1 \\
\hline 9 & 2402718 & 0 & 100 & 1 \\
\hline
\end{tabular}


(Continued) Supplemental Table 2. Bulked segregant analysis (BSA) based delta $(\Delta)$ single nucleotide polymorphism (SNP)_index (1.2-3.6 Mb) of chromosome 9; Standard type bulk is abbreviated by S-bulk and Dwarf type bulk is abbreviated by D-bulk.

\begin{tabular}{|c|c|c|c|c|}
\hline Chr. & Physical location & SNP index, S-bulk (\%) & SNP index, D-bulk (\%) & $\Delta \mathrm{SNP}$ index \\
\hline 9 & 2402932 & 3.7 & 100 & 0.963 \\
\hline 9 & 2403433 & 0 & 100 & 1 \\
\hline 9 & 2403861 & 0 & 100 & 1 \\
\hline 9 & 2403866 & 0 & 100 & 1 \\
\hline 9 & 2404535 & 0 & 100 & 1 \\
\hline 9 & 2406204 & 0 & 100 & 1 \\
\hline 9 & 2409130 & 0 & 100 & 1 \\
\hline 9 & 2409131 & 0 & 100 & 1 \\
\hline 9 & 2411116 & 3.12 & 100 & 0.9688 \\
\hline 9 & 2412868 & 0 & 33.33 & 0.3333 \\
\hline 9 & 2414760 & 3.7 & 100 & 0.963 \\
\hline 9 & 2415514 & 0 & 100 & 1 \\
\hline 9 & 2416625 & 0 & 100 & 1 \\
\hline 9 & 2416657 & 0 & 100 & 1 \\
\hline 9 & 2417258 & 0 & 100 & 1 \\
\hline 9 & 2417848 & 0 & 100 & 1 \\
\hline 9 & 2418283 & 0 & 100 & 1 \\
\hline 9 & 2418327 & 0 & 100 & 1 \\
\hline 9 & 2418616 & 0 & 93.33 & 0.9333 \\
\hline 9 & 2418678 & 0 & 100 & 1 \\
\hline 9 & 2419089 & 0 & 100 & 1 \\
\hline 9 & 2422062 & 0 & 100 & 1 \\
\hline 9 & 2422296 & 0 & 100 & 1 \\
\hline 9 & 2436281 & 0 & 92.31 & 0.9231 \\
\hline 9 & 2436459 & 0 & 100 & 1 \\
\hline 9 & 2439902 & 0 & 100 & 1 \\
\hline 9 & 2449945 & 0 & 100 & 1 \\
\hline 9 & 2451074 & 2.94 & 100 & 0.9706 \\
\hline 9 & 2451123 & 0 & 100 & 1 \\
\hline 9 & 2451217 & 0 & 100 & 1 \\
\hline 9 & 2452381 & 0 & 100 & 1 \\
\hline 9 & 2458540 & 3.57 & 100 & 0.9643 \\
\hline 9 & 2461691 & 0 & 100 & 1 \\
\hline 9 & 2461704 & 0 & 100 & 1 \\
\hline 9 & 2472995 & 0 & 100 & 1 \\
\hline 9 & 2477076 & 0 & 100 & 1 \\
\hline 9 & 2481459 & 0 & 100 & 1 \\
\hline 9 & 2482890 & 0 & 100 & 1 \\
\hline 9 & 2491383 & 100 & 0 & -1 \\
\hline 9 & 2491595 & 0 & 100 & 1 \\
\hline 9 & 2493666 & 100 & 0 & -1 \\
\hline 9 & 2502035 & 0 & 100 & 1 \\
\hline 9 & 2502066 & 0 & 20 & 0.2 \\
\hline 9 & 2502091 & 0 & 100 & 1 \\
\hline 9 & 2537037 & 0 & 20 & 0.2 \\
\hline 9 & 2537063 & 0 & 22.22 & 0.2222 \\
\hline 9 & 2537073 & 0 & 25 & 0.25 \\
\hline 9 & 2567072 & 0 & 20 & 0.2 \\
\hline 9 & 2567076 & 0 & 25 & 0.25 \\
\hline 9 & 2567112 & 0 & 25 & 0.25 \\
\hline 9 & 2567114 & 0 & 22.22 & 0.2222 \\
\hline 9 & 2567116 & 0 & 22.22 & 0.2222 \\
\hline 9 & 2567122 & 0 & 22.22 & 0.2222 \\
\hline 9 & 2567126 & 0 & 25 & 0.25 \\
\hline 9 & 2567133 & 0 & 22.22 & 0.2222 \\
\hline 9 & 2567135 & 0 & 22.22 & 0.2222 \\
\hline
\end{tabular}


(Continued) Supplemental Table 2. Bulked segregant analysis (BSA) based delta $(\Delta)$ single nucleotide polymorphism (SNP)_index (1.2-3.6 Mb) of chromosome 9; Standard type bulk is abbreviated by S-bulk and Dwarf type bulk is abbreviated by D-bulk.

\begin{tabular}{|c|c|c|c|c|}
\hline Chr. & Physical location & SNP index, S-bulk (\%) & SNP index, D-bulk (\%) & $\Delta \mathrm{SNP}$ index \\
\hline 9 & 2567162 & 0 & 20 & 0.2 \\
\hline 9 & 2567301 & 0 & 22.22 & 0.2222 \\
\hline 9 & 2567307 & 0 & 22.22 & 0.2222 \\
\hline 9 & 2567310 & 0 & 22.22 & 0.2222 \\
\hline 9 & 2567362 & 0 & 22.22 & 0.2222 \\
\hline 9 & 2584854 & 0 & 25 & 0.25 \\
\hline 9 & 2584866 & 0 & 23.08 & 0.2308 \\
\hline 9 & 2584875 & 0 & 33.33 & 0.3333 \\
\hline 9 & 2584880 & 0 & 33.33 & 0.3333 \\
\hline 9 & 2584881 & 0 & 30 & 0.3 \\
\hline 9 & 2584884 & 0 & 27.27 & 0.2727 \\
\hline 9 & 2584887 & 0 & 27.27 & 0.2727 \\
\hline 9 & 2584910 & 0 & 30 & 0.3 \\
\hline 9 & 2584929 & 0 & 30.77 & 0.3077 \\
\hline 9 & 2584947 & 0 & 25 & 0.25 \\
\hline 9 & 2610478 & 0 & 100 & 1 \\
\hline 9 & 2610694 & 0 & 100 & 1 \\
\hline 9 & 2616245 & 0 & 100 & 1 \\
\hline 9 & 2619319 & 94.44 & 100 & 0.0556 \\
\hline 9 & 2620436 & 96.55 & 100 & 0.0345 \\
\hline 9 & 2622847 & 88.24 & 80 & -0.0824 \\
\hline 9 & 2626097 & 94.44 & 100 & 0.0556 \\
\hline 9 & 2655180 & 9.52 & 60 & 0.5048 \\
\hline 9 & 2655193 & 45.45 & 71.43 & 0.2598 \\
\hline 9 & 2655230 & 86.36 & 100 & 0.1364 \\
\hline 9 & 2657543 & 100 & 91.67 & -0.0833 \\
\hline 9 & 2657645 & 0 & 22.22 & 0.2222 \\
\hline 9 & 2657648 & 0 & 22.22 & 0.2222 \\
\hline 9 & 2657652 & 0 & 25 & 0.25 \\
\hline 9 & 2657658 & 0 & 22.22 & 0.2222 \\
\hline 9 & 2657667 & 0 & 20 & 0.2 \\
\hline 9 & 2659869 & 95.65 & 100 & 0.0435 \\
\hline 9 & 2666625 & 0 & 25 & 0.25 \\
\hline 9 & 2666626 & 0 & 25 & 0.25 \\
\hline 9 & 2666652 & 0 & 25 & 0.25 \\
\hline 9 & 2666659 & 0 & 25 & 0.25 \\
\hline 9 & 2666662 & 0 & 28.57 & 0.2857 \\
\hline 9 & 2666665 & 0 & 25 & 0.25 \\
\hline 9 & 2666667 & 0 & 28.57 & 0.2857 \\
\hline 9 & 2678409 & 0 & 28.57 & 0.2857 \\
\hline 9 & 2723789 & 0 & 22.22 & 0.2222 \\
\hline 9 & 2723841 & 0 & 22.22 & 0.2222 \\
\hline 9 & 2723846 & 0 & 20 & 0.2 \\
\hline 9 & 2723856 & 0 & 23.08 & 0.2308 \\
\hline 9 & 2723905 & 0 & 20 & 0.2 \\
\hline 9 & 2726250 & 0 & 20 & 0.2 \\
\hline 9 & 2726277 & 0 & 27.27 & 0.2727 \\
\hline 9 & 2726322 & 0 & 20 & 0.2 \\
\hline 9 & 2726343 & 0 & 25 & 0.25 \\
\hline 9 & 2726352 & 0 & 23.08 & 0.2308 \\
\hline 9 & 2737274 & 84.62 & 0 & -0.8462 \\
\hline 9 & 2738918 & 100 & 0 & -1 \\
\hline 9 & 2739100 & 100 & 0 & -1 \\
\hline 9 & 2741260 & 100 & 0 & -1 \\
\hline 9 & 2741358 & 100 & 0 & -1 \\
\hline 9 & 2748178 & 100 & 0 & -1 \\
\hline
\end{tabular}


(Continued) Supplemental Table 2. Bulked segregant analysis (BSA) based delta $(\Delta)$ single nucleotide polymorphism (SNP)_index (1.2-3.6 Mb) of chromosome 9; Standard type bulk is abbreviated by S-bulk and Dwarf type bulk is abbreviated by D-bulk.

\begin{tabular}{|c|c|c|c|c|}
\hline Chr. & Physical location & SNP index, S-bulk (\%) & SNP index, D-bulk (\%) & $\Delta \mathrm{SNP}$ index \\
\hline 9 & 2748463 & 100 & 0 & -1 \\
\hline 9 & 2748504 & 100 & 0 & -1 \\
\hline 9 & 2753455 & 100 & 0 & -1 \\
\hline 9 & 2756855 & 94.44 & 0 & -0.9444 \\
\hline 9 & 2760175 & 100 & 0 & -1 \\
\hline 9 & 2760311 & 100 & 0 & -1 \\
\hline 9 & 2760560 & 100 & 0 & -1 \\
\hline 9 & 2763456 & 100 & 0 & -1 \\
\hline 9 & 2766557 & 100 & 0 & -1 \\
\hline 9 & 2767793 & 0 & 30 & 0.3 \\
\hline 9 & 2767803 & 0 & 27.27 & 0.2727 \\
\hline 9 & 2767805 & 0 & 30 & 0.3 \\
\hline 9 & 2767817 & 0 & 25 & 0.25 \\
\hline 9 & 2767835 & 0 & 27.27 & 0.2727 \\
\hline 9 & 2767848 & 0 & 27.27 & 0.2727 \\
\hline 9 & 2770464 & 100 & 14.29 & -0.8571 \\
\hline 9 & 2770680 & 0 & 22.22 & 0.2222 \\
\hline 9 & 2770787 & 0 & 20 & 0.2 \\
\hline 9 & 2771028 & 100 & 11.11 & -0.8889 \\
\hline 9 & 2771051 & 0 & 25 & 0.25 \\
\hline 9 & 2771291 & 0 & 22.22 & 0.2222 \\
\hline 9 & 2773645 & 100 & 0 & -1 \\
\hline 9 & 2774299 & 100 & 0 & -1 \\
\hline 9 & 2774342 & 100 & 0 & -1 \\
\hline 9 & 2774385 & 100 & 0 & -1 \\
\hline 9 & 2774739 & 100 & 0 & -1 \\
\hline 9 & 2778504 & 21.43 & 100 & 0.7857 \\
\hline 9 & 2785650 & 95.83 & 0 & -0.9583 \\
\hline 9 & 2786203 & 100 & 0 & -1 \\
\hline 9 & 2787131 & 0 & 100 & 1 \\
\hline 9 & 2790008 & 0 & 25 & 0.25 \\
\hline 9 & 2790026 & 0 & 28.57 & 0.2857 \\
\hline 9 & 2790047 & 0 & 25 & 0.25 \\
\hline 9 & 2790050 & 0 & 25 & 0.25 \\
\hline 9 & 2790125 & 0 & 25 & 0.25 \\
\hline 9 & 2790269 & 0 & 22.22 & 0.2222 \\
\hline 9 & 2790281 & 0 & 22.22 & 0.2222 \\
\hline 9 & 2790301 & 0 & 22.22 & 0.2222 \\
\hline 9 & 2798739 & 0 & 25 & 0.25 \\
\hline 9 & 2798764 & 0 & 20 & 0.2 \\
\hline 9 & 2798771 & 0 & 22.22 & 0.2222 \\
\hline 9 & 2798809 & 0 & 20 & 0.2 \\
\hline 9 & 2800283 & 0 & 100 & 1 \\
\hline 9 & 2818671 & 0 & 22.22 & 0.2222 \\
\hline 9 & 2818676 & 0 & 20 & 0.2 \\
\hline 9 & 2825486 & 0 & 100 & 1 \\
\hline 9 & 2828142 & 0 & 100 & 1 \\
\hline 9 & 2832708 & 0 & 100 & 1 \\
\hline 9 & 2836103 & 3.03 & 100 & 0.9697 \\
\hline 9 & 2851824 & 0 & 100 & 1 \\
\hline 9 & 2852517 & 0 & 100 & 1 \\
\hline 9 & 2854884 & 0 & 100 & 1 \\
\hline 9 & 2854904 & 0 & 100 & 1 \\
\hline 9 & 2856781 & 0 & 100 & 1 \\
\hline 9 & 2857379 & 0 & 100 & 1 \\
\hline 9 & 2864593 & 0 & 25 & 0.25 \\
\hline
\end{tabular}

(Continued on next page) 
(Continued) Supplemental Table 2. Bulked segregant analysis (BSA) based delta $(\Delta)$ single nucleotide polymorphism (SNP)_index (1.2-3.6 Mb) of chromosome 9; Standard type bulk is abbreviated by S-bulk and Dwarf type bulk is abbreviated by D-bulk.

\begin{tabular}{|c|c|c|c|c|}
\hline Chr. & Physical location & SNP index, S-bulk (\%) & SNP index, D-bulk (\%) & $\Delta \mathrm{SNP}$ index \\
\hline 9 & 2864608 & 0 & 25 & 0.25 \\
\hline 9 & 2864609 & 0 & 25 & 0.25 \\
\hline 9 & 2864627 & 0 & 20 & 0.2 \\
\hline 9 & 2864640 & 0 & 20 & 0.2 \\
\hline 9 & 2864654 & 0 & 20 & 0.2 \\
\hline 9 & 2864702 & 0 & 20 & 0.2 \\
\hline 9 & 2867575 & 0 & 22.22 & 0.2222 \\
\hline 9 & 2887049 & 0 & 100 & 1 \\
\hline 9 & 2894527 & 3.03 & 28.57 & 0.2554 \\
\hline 9 & 2894548 & 6.67 & 35.71 & 0.2904 \\
\hline 9 & 2894558 & 20.83 & 0 & -0.2083 \\
\hline 9 & 2894561 & 74.47 & 60 & -0.1447 \\
\hline 9 & 2894574 & 29.79 & 26.67 & -0.0312 \\
\hline 9 & 2894584 & 51.16 & 66.67 & 0.1551 \\
\hline 9 & 2894622 & 6.06 & 27.27 & 0.2121 \\
\hline 9 & 2894641 & 54.17 & 54.55 & 0.0038 \\
\hline 9 & 2894703 & 62.96 & 54.55 & -0.0841 \\
\hline 9 & 2894747 & 60 & 70 & 0.1 \\
\hline 9 & 2894791 & 30.77 & 35 & 0.0423 \\
\hline 9 & 2894853 & 0 & 22.22 & 0.2222 \\
\hline 9 & 2894872 & 0 & 26.32 & 0.2632 \\
\hline 9 & 2894879 & 45.24 & 25 & -0.2024 \\
\hline 9 & 2894912 & 41.46 & 57.14 & 0.1568 \\
\hline 9 & 2894913 & 37.21 & 21.43 & -0.1578 \\
\hline 9 & 2894925 & 88.1 & 75 & -0.131 \\
\hline 9 & 2894943 & 90.24 & 81.82 & -0.0842 \\
\hline 9 & 2920537 & 0 & 20 & 0.2 \\
\hline 9 & 2933322 & 0 & 100 & 1 \\
\hline 9 & 2933369 & 4.55 & 100 & 0.9545 \\
\hline 9 & 2933600 & 0 & 100 & 1 \\
\hline 9 & 2934105 & 0 & 22.22 & 0.2222 \\
\hline 9 & 2934109 & 0 & 20 & 0.2 \\
\hline 9 & 2936387 & 0 & 100 & 1 \\
\hline 9 & 2956997 & 0 & 100 & 1 \\
\hline 9 & 2963894 & 0 & 100 & 1 \\
\hline 9 & 2972300 & 0 & 20 & 0.2 \\
\hline 9 & 2972323 & 0 & 20 & 0.2 \\
\hline 9 & 2972329 & 0 & 22.22 & 0.2222 \\
\hline 9 & 2976491 & 100 & 11.11 & -0.8889 \\
\hline 9 & 2985402 & 0 & 100 & 1 \\
\hline 9 & 2986767 & 0 & 50 & 0.5 \\
\hline 9 & 2986773 & 0 & 50 & 0.5 \\
\hline 9 & 2986780 & 0 & 44.44 & 0.4444 \\
\hline 9 & 2997880 & 0 & 25 & 0.25 \\
\hline 9 & 2997886 & 0 & 25 & 0.25 \\
\hline 9 & 2997895 & 0 & 25 & 0.25 \\
\hline 9 & 3004751 & 100 & 0 & -1 \\
\hline 9 & 3004897 & 100 & 12.5 & -0.875 \\
\hline 9 & 3009985 & 0 & 25 & 0.25 \\
\hline 9 & 3010011 & 0 & 25 & 0.25 \\
\hline 9 & 3010049 & 0 & 20 & 0.2 \\
\hline 9 & 3011846 & 0 & 25 & 0.25 \\
\hline 9 & 3011862 & 0 & 25 & 0.25 \\
\hline 9 & 3011869 & 0 & 22.22 & 0.2222 \\
\hline 9 & 3011870 & 0 & 22.22 & 0.2222 \\
\hline 9 & 3011902 & 0 & 25 & 0.25 \\
\hline
\end{tabular}


(Continued) Supplemental Table 2. Bulked segregant analysis (BSA) based delta $(\Delta)$ single nucleotide polymorphism (SNP)_index (1.2-3.6 Mb) of chromosome 9; Standard type bulk is abbreviated by S-bulk and Dwarf type bulk is abbreviated by D-bulk.

\begin{tabular}{|c|c|c|c|c|}
\hline Chr. & Physical location & SNP index, S-bulk (\%) & SNP index, D-bulk (\%) & $\Delta \mathrm{SNP}$ index \\
\hline 9 & 3014615 & 0 & 100 & 1 \\
\hline 9 & 3014648 & 0 & 100 & 1 \\
\hline 9 & 3016027 & 100 & 0 & -1 \\
\hline 9 & 3016201 & 100 & 0 & -1 \\
\hline 9 & 3016245 & 3.7 & 100 & 0.963 \\
\hline 9 & 3016281 & 0 & 100 & 1 \\
\hline 9 & 3016340 & 0 & 100 & 1 \\
\hline 9 & 3016420 & 0 & 100 & 1 \\
\hline 9 & 3016480 & 0 & 100 & 1 \\
\hline 9 & 3024889 & 0 & 25 & 0.25 \\
\hline 9 & 3024893 & 0 & 22.22 & 0.2222 \\
\hline 9 & 3058246 & 0 & 20 & 0.2 \\
\hline 9 & 3069362 & 0 & 25 & 0.25 \\
\hline 9 & 3075943 & 0 & 87.5 & 0.875 \\
\hline 9 & 3076048 & 4.55 & 87.5 & 0.8295 \\
\hline 9 & 3084730 & 0 & 100 & 1 \\
\hline 9 & 3085204 & 0 & 88.89 & 0.8889 \\
\hline 9 & 3085409 & 0 & 100 & 1 \\
\hline 9 & 3086216 & 0 & 100 & 1 \\
\hline 9 & 3086442 & 3.57 & 100 & 0.9643 \\
\hline 9 & 3089201 & 0 & 100 & 1 \\
\hline 9 & 3090682 & 0 & 100 & 1 \\
\hline 9 & 3092534 & 0 & 100 & 1 \\
\hline 9 & 3092772 & 0 & 100 & 1 \\
\hline 9 & 3096393 & 0 & 100 & 1 \\
\hline 9 & 3101682 & 0 & 100 & 1 \\
\hline 9 & 3102584 & 0 & 100 & 1 \\
\hline 9 & 3102706 & 0 & 55.56 & 0.5556 \\
\hline 9 & 3102754 & 0 & 88.89 & 0.8889 \\
\hline 9 & 3104321 & 0 & 100 & 1 \\
\hline 9 & 3104421 & 0 & 100 & 1 \\
\hline 9 & 3105042 & 0 & 100 & 1 \\
\hline 9 & 3105266 & 3.57 & 100 & 0.9643 \\
\hline 9 & 3106295 & 0 & 100 & 1 \\
\hline 9 & 3106920 & 0 & 100 & 1 \\
\hline 9 & 3106943 & 0 & 100 & 1 \\
\hline 9 & 3107116 & 0 & 100 & 1 \\
\hline 9 & 3107174 & 0 & 100 & 1 \\
\hline 9 & 3107185 & 0 & 100 & 1 \\
\hline 9 & 3107412 & 0 & 100 & 1 \\
\hline 9 & 3108417 & 0 & 100 & 1 \\
\hline 9 & 3108447 & 0 & 100 & 1 \\
\hline 9 & 3108571 & 0 & 100 & 1 \\
\hline 9 & 3108914 & 0 & 100 & 1 \\
\hline 9 & 3123529 & 0 & 100 & 1 \\
\hline 9 & 3125363 & 0 & 100 & 1 \\
\hline 9 & 3125873 & 0 & 87.5 & 0.875 \\
\hline 9 & 3125891 & 0 & 20 & 0.2 \\
\hline 9 & 3137556 & 0 & 100 & 1 \\
\hline 9 & 3139711 & 0 & 28.57 & 0.2857 \\
\hline 9 & 3139723 & 0 & 28.57 & 0.2857 \\
\hline 9 & 3139758 & 0 & 25 & 0.25 \\
\hline 9 & 3146397 & 0 & 20 & 0.2 \\
\hline 9 & 3146401 & 0 & 20 & 0.2 \\
\hline 9 & 3146402 & 0 & 20 & 0.2 \\
\hline 9 & 3146469 & 0 & 22.22 & 0.2222 \\
\hline
\end{tabular}

(Continued on next page) 
(Continued) Supplemental Table 2. Bulked segregant analysis (BSA) based delta $(\Delta)$ single nucleotide polymorphism (SNP)_index (1.2-3.6 Mb) of chromosome 9; Standard type bulk is abbreviated by S-bulk and Dwarf type bulk is abbreviated by D-bulk.

\begin{tabular}{|c|c|c|c|c|}
\hline Chr. & Physical location & SNP index, S-bulk (\%) & SNP index, D-bulk (\%) & $\Delta \mathrm{SNP}$ index \\
\hline 9 & 3153471 & 0 & 100 & 1 \\
\hline 9 & 3153502 & 0 & 100 & 1 \\
\hline 9 & 3155329 & 0 & 100 & 1 \\
\hline 9 & 3168811 & 2.94 & 100 & 0.9706 \\
\hline 9 & 3169434 & 0 & 100 & 1 \\
\hline 9 & 3171493 & 0 & 91.67 & 0.9167 \\
\hline 9 & 3173536 & 0 & 100 & 1 \\
\hline 9 & 3174760 & 0 & 22.22 & 0.2222 \\
\hline 9 & 3180061 & 0 & 100 & 1 \\
\hline 9 & 3185633 & 0 & 100 & 1 \\
\hline 9 & 3186695 & 0 & 100 & 1 \\
\hline 9 & 3189441 & 0 & 100 & 1 \\
\hline 9 & 3191135 & 0 & 25 & 0.25 \\
\hline 9 & 3191137 & 0 & 20 & 0.2 \\
\hline 9 & 3191138 & 0 & 25 & 0.25 \\
\hline 9 & 3191157 & 0 & 20 & 0.2 \\
\hline 9 & 3191161 & 0 & 20 & 0.2 \\
\hline 9 & 3191172 & 0 & 20 & 0.2 \\
\hline 9 & 3199055 & 0 & 100 & 1 \\
\hline 9 & 3201870 & 0 & 100 & 1 \\
\hline 9 & 3210003 & 0 & 100 & 1 \\
\hline 9 & 3223359 & 0 & 20 & 0.2 \\
\hline 9 & 3223372 & 0 & 22.22 & 0.2222 \\
\hline 9 & 3223374 & 0 & 20 & 0.2 \\
\hline 9 & 3223375 & 0 & 20 & 0.2 \\
\hline 9 & 3223376 & 100 & 80 & -0.2 \\
\hline 9 & 3226356 & 0 & 20 & 0.2 \\
\hline 9 & 3231208 & 0 & 25 & 0.25 \\
\hline 9 & 3231209 & 0 & 25 & 0.25 \\
\hline 9 & 3231210 & 0 & 28.57 & 0.2857 \\
\hline 9 & 3231332 & 0 & 28.57 & 0.2857 \\
\hline 9 & 3231341 & 0 & 22.22 & 0.2222 \\
\hline 9 & 3231347 & 0 & 22.22 & 0.2222 \\
\hline 9 & 3231355 & 0 & 20 & 0.2 \\
\hline 9 & 3231363 & 0 & 25 & 0.25 \\
\hline 9 & 3231364 & 0 & 22.22 & 0.2222 \\
\hline 9 & 3231365 & 0 & 20 & 0.2 \\
\hline 9 & 3261784 & 21.43 & 37.5 & 0.1607 \\
\hline 9 & 3261789 & 22.22 & 42.86 & 0.2064 \\
\hline 9 & 3277805 & 0 & 100 & 1 \\
\hline 9 & 3277911 & 8.33 & 100 & 0.9167 \\
\hline 9 & 3280952 & 0 & 90.91 & 0.9091 \\
\hline 9 & 3288965 & 0 & 100 & 1 \\
\hline 9 & 3290889 & 0 & 100 & 1 \\
\hline 9 & 3291546 & 0 & 100 & 1 \\
\hline 9 & 3292155 & 0 & 100 & 1 \\
\hline 9 & 3293090 & 0 & 100 & 1 \\
\hline 9 & 3298285 & 0 & 20 & 0.2 \\
\hline 9 & 3298995 & 0 & 20 & 0.2 \\
\hline 9 & 3302787 & 0 & 66.67 & 0.6667 \\
\hline 9 & 3319944 & 100 & 0 & -1 \\
\hline 9 & 3329693 & 100 & 92.86 & -0.0714 \\
\hline 9 & 3336080 & 0 & 100 & 1 \\
\hline 9 & 3336376 & 0 & 100 & 1 \\
\hline 9 & 3337337 & 0 & 22.22 & 0.2222 \\
\hline 9 & 3337392 & 0 & 20 & 0.2 \\
\hline
\end{tabular}


(Continued) Supplemental Table 2. Bulked segregant analysis (BSA) based delta $(\Delta)$ single nucleotide polymorphism (SNP)_index (1.2-3.6 Mb) of chromosome 9; Standard type bulk is abbreviated by S-bulk and Dwarf type bulk is abbreviated by D-bulk.

\begin{tabular}{|c|c|c|c|c|}
\hline Chr. & Physical location & SNP index, S-bulk (\%) & SNP index, D-bulk (\%) & $\Delta \mathrm{SNP}$ index \\
\hline 9 & 3337792 & 0 & 100 & 1 \\
\hline 9 & 3338233 & 2.86 & 100 & 0.9714 \\
\hline 9 & 3340174 & 0 & 22.22 & 0.2222 \\
\hline 9 & 3340188 & 0 & 22.22 & 0.2222 \\
\hline 9 & 3341540 & 0 & 100 & 1 \\
\hline 9 & 3347836 & 0 & 100 & 1 \\
\hline 9 & 3352452 & 42.86 & 20 & -0.2286 \\
\hline 9 & 3358561 & 0 & 22.22 & 0.2222 \\
\hline 9 & 3367932 & 0 & 28.57 & 0.2857 \\
\hline 9 & 3377486 & 0 & 100 & 1 \\
\hline 9 & 3382357 & 0 & 100 & 1 \\
\hline 9 & 3382610 & 0 & 100 & 1 \\
\hline 9 & 3383908 & 0 & 100 & 1 \\
\hline 9 & 3396453 & 0 & 100 & 1 \\
\hline 9 & 3399890 & 0 & 100 & 1 \\
\hline 9 & 3401153 & 0 & 100 & 1 \\
\hline 9 & 3402087 & 0 & 100 & 1 \\
\hline 9 & 3402892 & 0 & 100 & 1 \\
\hline 9 & 3410514 & 7.14 & 100 & 0.9286 \\
\hline 9 & 3424964 & 0 & 100 & 1 \\
\hline 9 & 3425040 & 4 & 100 & 0.96 \\
\hline 9 & 3425049 & 0 & 100 & 1 \\
\hline 9 & 3425257 & 0 & 100 & 1 \\
\hline 9 & 3425302 & 0 & 100 & 1 \\
\hline 9 & 3425565 & 0 & 100 & 1 \\
\hline 9 & 3425969 & 0 & 100 & 1 \\
\hline 9 & 3426749 & 0 & 100 & 1 \\
\hline 9 & 3430922 & 0 & 30 & 0.3 \\
\hline 9 & 3430940 & 0 & 28.57 & 0.2857 \\
\hline 9 & 3431065 & 0 & 25 & 0.25 \\
\hline 9 & 3431074 & 0 & 25 & 0.25 \\
\hline 9 & 3431078 & 0 & 25 & 0.25 \\
\hline 9 & 3431183 & 0 & 33.33 & 0.3333 \\
\hline 9 & 3431189 & 0 & 25 & 0.25 \\
\hline 9 & 3434854 & 0 & 100 & 1 \\
\hline 9 & 3435259 & 0 & 87.5 & 0.875 \\
\hline 9 & 3440961 & 0 & 100 & 1 \\
\hline 9 & 3441177 & 0 & 100 & 1 \\
\hline 9 & 3441862 & 0 & 100 & 1 \\
\hline 9 & 3445309 & 0 & 100 & 1 \\
\hline 9 & 3445415 & 0 & 100 & 1 \\
\hline 9 & 3448723 & 0 & 100 & 1 \\
\hline 9 & 3451949 & 31.58 & 33.33 & 0.0175 \\
\hline 9 & 3454202 & 0 & 100 & 1 \\
\hline 9 & 3455878 & 0 & 100 & 1 \\
\hline 9 & 3457311 & 0 & 100 & 1 \\
\hline 9 & 3457476 & 0 & 22.22 & 0.2222 \\
\hline 9 & 3457478 & 0 & 22.22 & 0.2222 \\
\hline 9 & 3457482 & 0 & 22.22 & 0.2222 \\
\hline 9 & 3457499 & 0 & 37.5 & 0.375 \\
\hline 9 & 3457500 & 0 & 33.33 & 0.3333 \\
\hline 9 & 3457509 & 0 & 33.33 & 0.3333 \\
\hline 9 & 3457535 & 0 & 28.57 & 0.2857 \\
\hline 9 & 3458053 & 0 & 100 & 1 \\
\hline 9 & 3459488 & 0 & 22.22 & 0.2222 \\
\hline 9 & 3459507 & 0 & 22.22 & 0.2222 \\
\hline
\end{tabular}


(Continued) Supplemental Table 2. Bulked segregant analysis (BSA) based delta $(\Delta)$ single nucleotide polymorphism (SNP)_index (1.2-3.6 Mb) of chromosome 9; Standard type bulk is abbreviated by S-bulk and Dwarf type bulk is abbreviated by D-bulk.

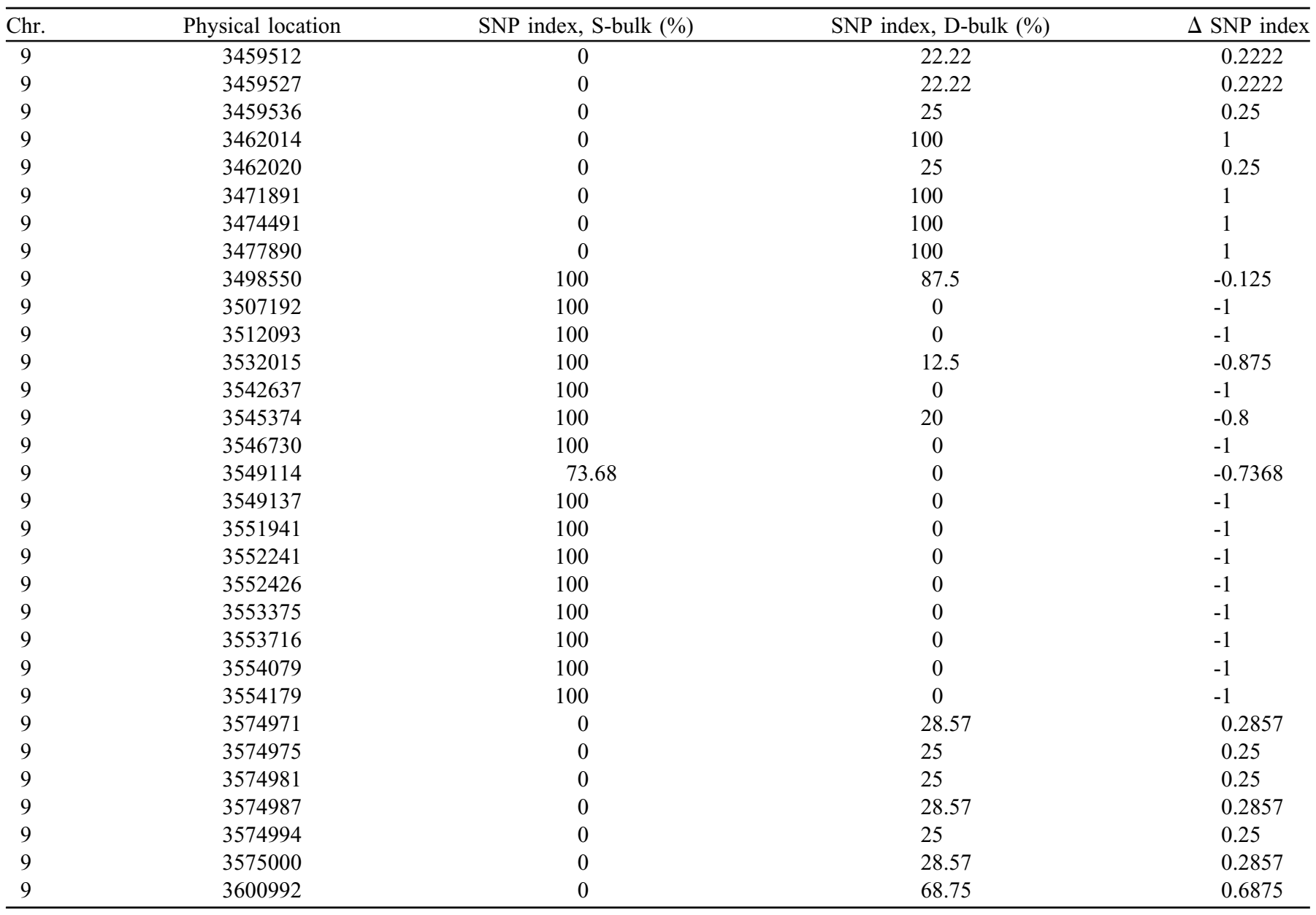

